\title{
Strategy Development for Special Operations Force Logistics
}

\section{David M Moore, David Allen and Peter D Antill}

The aim of this paper is to highlight the specific logistics problems which Special Operations Forces have, given their highly specialist nature and the factors that act upon them such as external (political, economic and national culture) and internal influences as well as the changing face of warfare. It will examine the ways in which Special Operations Forces are currently supported logistically and will go on to consider the ways by which commercial 'best-practice' could be used to enhance the mission effectiveness of forces employed in this role.

\section{Introduction}

Special Operations Forces (SOF) or Special Forces have traditionally invoked a degree of romantic heroism. The images of Lawrence of Arabia, David Stirling's Long-Range Desert Group, Merrill's Marauders, Otto Skorzeny's rescue of Mussolini in 1943, and the SAS exploits with the Iranian Embassy and in the Persian Gulf War (Bravo Two-Zero) have filled us with awe and wonder. While this has fostered an ever-growing interest and body of literature around these sorts of forces and their operations, such matters should really be viewed as belonging to the realm of serious military operations rather than undercover romantic heroism that is the stuff of films such as the James Bond series or True Lies. 
There are as many definitions of Special Operations as there are states that have the forces to carry them out. Most share common elements though in that they are operations that are more frequently influenced by considerations at a higher level than usual, and require "oversight at the national level. Special Operations differ from conventional operations in the degree of physical and political risk"1, which indicates a greater degree of political influence from national command authorities. Typically, SOF are set apart from the rest of the armed forces and are considered elite forces that are "specifically organised, trained and equipped to conduct or support insurgency, sabotage, psychological, deception, counter-terrorist, foreign assistance or commando-type operations". ${ }^{2}$

Both SOF and the rest of the armed forces have had to come to terms with the end of the Cold War, which has led to the contraction of defence budgets across much of the globe, especially in respect to NATO and the former Warsaw Pact. This has meant fewer resources with which to procure equipment and conduct operations and many countries have downsized their armed forces and turned to streamlining the logistic support given to those forces. In many cases this has led to a greater role for third-party contractors. The military have examined the steps taken by commercial companies in the past few decades in an attempt to find out if commercial 'bestpractice' can be applied to the logistic support for their armed forces and make scarce resources stretch that much farther. 


\section{Special Operations Forces}

While many SOF have unique identities and histories, there are a number of common threads that apply to most of them. A military elite is defined by three criteria: ${ }^{3}$

- It must be perpetually assigned to special missions.

- It conducts missions requiring only a few highly trained personnel.

- They have a reputation for bravery and success.

There are four areas where SOF can be contrasted with other forces:

- The roles they are to undertake.

- The resources they are given with which to undertake their roles.

- The characteristics of the personnel assigned to these forces.

- The nature of the control exerted on these forces.

Most SOF declare that their roles are different from all others and this has to do with political, cultural and historical factors more than military requirements, but the majority of SOF roles can be fitted into one of three areas. ${ }^{4}$ The first area contains the traditional combat missions, such as Special Reconnaissance in the enemy rear and Offensive Action against targets of strategic or operational importance. It may also include search and rescue of downed aircrew or isolated friendly forces (such as the SAS operation to free members of the Royal Irish Regiment in Sierra Leone). The second area has gained a new interest in many quarters, particularly the United States, that of Low Intensity Conflict (LIC) or Operations Other Than War (OOTW). This term covers those conflicts that are generally between states or groups within a 
state and that are below the intensity associated with conventional conflict but above the 'normal' peacetime routine. Such conflicts are generally localised ones, often in the less developed regions of the world, but quite often have security implications. SOF roles in this sort of conflict would include counter-insurgency (COIN) support and influence $(\mathrm{SI})$ to indigenous forces, combating organised crime, counterterrorism (CT) and psychological operations (PsyOps). The third area is in many ways supplemental to LIC operations and that is 'peace operations' in support of civilian authorities. This includes duties such as Close Protection Teams for VIPs (CPT), negotiation team escorts, the arrest of persons indicted for war crimes (PIFWC) and establishing relations in crisis areas. It must be remembered however that there is considerable overlap between the three areas as many missions and activities cannot always be neatly categorised.

Resource allocation is usually indicative of the political and military commitment to SOF, and includes not only financial commitment but also the political commitment to commit conventional forces to support SOF. Few nations have the resources to form large SOF groups (in NATO parlance, a Task Force) that can carry out operations with a high degree of self-sufficiency. In practice a Combined Joint Special Operations Task Force (CJSOTF) may be formed that can allocate scarce resources and improve co-ordination.

Personnel within these organisations who carry out missions are usually called Special Operators to distinguish themselves from those who support them. Such individuals usually have a high degree of motivation to accept the risks involved and are mainly volunteers although this is not a prerequisite. The character of an 
organisation can be defined by the career path for both enlisted men and officers and it may be that all personnel have to return to their parent units after completing a tour within the SOF.

Control of SOF can be decisive in determining its likely effectiveness, and also the resources allocated to operations. They can be controlled centrally alongside other state intelligence assets and agencies, or control can be devolved down to an operational commander.

SOF can be categorised and divided into three models, based on their characteristics, which correspond to the British, Russian and US SOF. The Comprehensive Qualitative Model (CQM) represents the model followed by the UK and is characterised by force of highly trained, but broadly skilled individuals with a degree of central control, a high degree of political commitment, but are only modestly resourced in comparison to other states. The Functional Quantitative Model (FQM) represents the earliest development of such commando-style forces and is a model still followed by the Russians. It is characterised by relatively large numbers of personnel who are trained on a narrow base and controlled by a wide variety of agencies. The Technical Functional Model (TFM) represents the course taken by the United States and is characterised by relatively large numbers which collectively cover a wide range of skills but are individually more narrowly trained than the CQM. There is quite a strong-degree of central control and political commitment but the size of the organisation (and the subsequent bureaucracy) is often a source of inter-service rivalry. 
The SOF of the UK, USA and Russia and therefore the different models, are borne out of different historical situations and development. The British SOF were created during the darkest moments in World War Two and have remained small and secretive with a high degree of political commitment but have lacked resources. This has led to them becoming highly flexible, as they have had to expect a lack of resources and have aimed to plan around that. The Russian SOF was born during the Revolution and has remained a secret organisation with its base spread across the state apparatus. They are relatively large, but lack somewhat in terms of resources and are less protective of their personnel. The US SOF has finally come out of its post-Vietnam depression and the failure of Operation Eagle Claw with the relative successes of Operations Urgent Fury ${ }^{5}$ (despite some embarrassing moments) and Just Cause ${ }^{6}$, and the highpoints of Operation Desert Shield / Storm and the conflict in the former Yugoslavia. They have a strong influence, have strong political commitment but represent a large bureaucracy.

In commercial terms, the UK SOF concentrate on their core competence of special operations and outsource specialist functions and support to other parts of the UK Armed Forces such as the Royal Signals, Royal Air Force or Army Air Corps. Other assets are then contracted in as required, although this raises the possibility that the third-party provider may have other priorities in conflict with those of the SOF. The US SOF represents a large corporation that is vertically integrated with a large number of shareholders. Whilst it controls its own assets, the shareholders often disagree on company policy and it is in danger of assuming a monolithic corporate ethos. Russian SOF could be seen as a gradually declining nationalised industry with a proud past but faces a lack of resources and strategic drift. Its stakeholders 
tend to be very possessive about their part of the corporation and little enthusiasm to become 'lean' and 'agile'.

\section{Factors Influencing the Way in which SOF Operate}

SOF do not operate in a vacuum. They are subject to the external forces of politics, economics and national culture (which is influenced by national history), internal forces within the SOF themselves, and the changing nature of war in the modern world.

One of the central influences on future SOF operations (and hence their demand for logistic support) is the rapidly changing nature of war. The end of the Cold War and the East-West balance has removed many of the old certainties and has meant an increase in the opportunities for SOF to be used. Many armed forces are now looking to answer the question, "who or what constitutes the threat?" It has been argued that an increasingly grey area " ... where control has shifted from legitimate governments to new half-political, half-criminal powers" ${ }^{7}$ is becoming the new threat. Such organisations, forces or entities are likely to grow out of environments where law and order is weak or non-existent, such as a failed state and it is possible that these activities will be sponsored by pariah states in an environment that is part-war and part-crime. The future of war is likely to the illegitimate child of Bosnia, Rwanda and Somalia, rather than the son of Desert Storm. ${ }^{8}$

The previous symmetry of war (where two combatants fight it out on the same level

of intensity and bound by similar rules) no longer exists. Planning for traditional 
warfare was relatively easy (such as in planning for a Warsaw Pact assault against Western Europe) but with the end of the Cold War and the traditional certainties having been swept away, this area of uncertainty has created the greatest pressure for both the regular armed forces and SOF to develop new methods of operation.

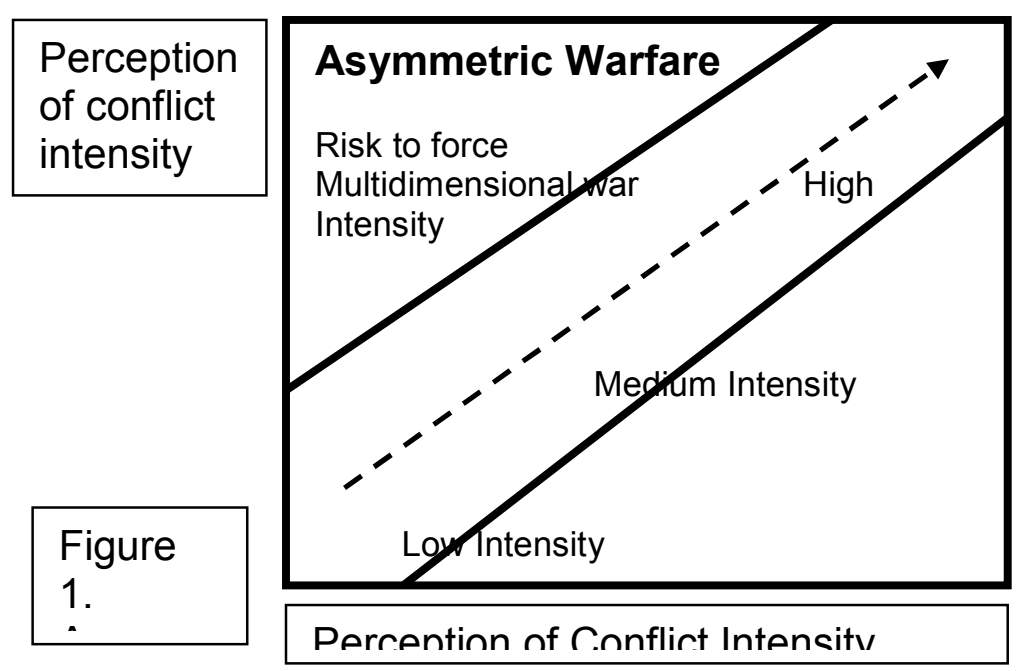

Figure 1 indicates the problems of warfighting in the post-Cold War era. If both sides' perceptions on the conflict, the risks involved and the intensity by which they are going to wage it, are roughly similar the conflict is going to be roughly symmetrical. This is shown by the central 'corridor' within the diagram, and conforms to the traditional notions of low, medium and high intensity. If however their perceptions are different then it is likely that asymmetrical conflict will take place. If one's own perception is too low, then there may be risks to the force involved in the operation, as it has insufficient resources either in terms of combat capability, logistic support or intelligence gathering (which is linked to the problems of multidimensional warfare). If one's own perception is greater then there is a risk of overreaction, of using too great a force, and handing the opponent a political victory. In the new 
post-Cold War era, SOF forces are most likely to operate in the areas either side of the 'corridor' either in support of a conventional force or on their own. The tragic events in New York on September 112001 have highlighted this and the impact asymmetric warfare can have. The terrorists effectively circumvented the US North American Air Defence (NORAD) network by hijacking internal domestic flights and proceeded to use them as flying bombs, while the subsequent operations in Afghanistan have featured the large-scale use of SOF.

A related concept to that of asymmetry is that of multidimensional warfare. Armed forces have, for most of the last one hundred years, operated in the four dimensions of the human senses ${ }^{9}$ but have increasingly sought to exploit the space that is beyond the human senses, known as cyberspace. ${ }^{10}$ As an example, some of the Somali warlords used a form of spatial warping against the US forces by moving freely without bodyguards or protection. They effectively became stealthy by not conforming to the US expectations of behaviour. The Americans did not expect such a major leader such as Mohamed Aidid to move about among the population in Mogadishu without escort and so was 'beyond their senses' even though he moved in clear daylight. This is known as spatial expansion, while spatial contraction links individuals via technology and so a major computer system can be attacked from anywhere in the world by effectively shrinking space and time.

Armed forces are therefore increasingly faced by enemies who are not bound by the norms of warfare and who are likely to blur the distinction between combatant and non-combatant. With the demonstrations of combat power in both the Gulf War and over Kosovo and Serbia, many smaller states are likely to realise that they cannot 
take on the might of either NATO or the United States in direct conflict and hope to win and therefore they will most probably choose different methods of warfare with which they might stand a better chance, such as economic warfare, terrorism, and media manipulation. In the post-Cold War world, the large conventional and nuclear forces that both East and West have built up may become increasingly impotent and it will increasingly be the SOF that can counteract the new threats.

How those SOF are employed is defined by the state's history. For example, the US sees the SOF as guardians of the pioneering tradition in the footsteps of the frontiersmen. ${ }^{11}$ France and Israel see SOF much more in the Machiavellian tradition and any transgression of International Law would have to be extremely serious to force them to act or apologise. The sinking of the Rainbow Warrior by French SOF in Auckland Harbour only led to a minor apology. ${ }^{12}$

SOF will also be affected by external forces. These can be categorised as political, economic and cultural:

- Political - As Clausewitz recognised long ago, it is "politics which beget war. Politics represents the intelligence, war merely its instrument, not the other way round. The only reasonable course in war is to subordinate the military viewpoint to the political."13 SOF are usually under tighter political control than the remainder of the armed forces. This control often brings with it political pressures that manifest themselves in, for example, trying to do more than they are reasonably capable of doing for domestic political reasons. Operation Eagle Claw $^{14}$ for example, was an attempt to free the US diplomatic hostages held in Tehran, Iran in 1980. While the President's National Security Advisor persuaded 
Jimmy Carter it could work, the intelligence officer of Delta Force, Wade Ishimoto, was less optimistic - "I didn't think it had a chance of succeeding."15 The mission was aborted after a number of the US Marine Corps helicopters had developed faults, which made the mission extremely risky, if not impossible. In the withdrawal, 8 US personnel were killed when an RH-53D helicopter collided with an MC-130 aircraft. Political demands for action had led to a mission that should not have taken place, or have taken place with much greater planning, preparation and care. Political pressures can place impossible demands or impose intolerable constraints. The SOF will have to manage this reality as best they can. SOF, as with most organisations, must adapt to the changing political and strategic conditions of the time in order to maximise their chances for operational success. It is important for the SOF that the politicians who have ultimate political authority are educated consumers if the SOF are not to be misused and face disaster, as is the case with the Brandenburgers ${ }^{16}$ and the Spetsnaz in Grosny.

- Economics - economics and politics are interlinked, especially in Western democracies where voters have a mandate on the performance of those in Government. Since the end of the Cold War there has been pressure on the defence budget to be reduced so the extra resources can be spent on other areas of the public sector. ${ }^{17}$ This is especially so for SOF which are seen as a 'gold-plated' capability with their specialist training, equipment and increased salaries. ${ }^{18}$ The pressure to demonstrate 'value for money' is especially acute, as many of the SOF's outputs can be hard to see, as they are indirect effects, or have to remain classified. Initiatives such as Project Capital in the UK (which 
aims to encourage the reduction of asset holding by charging budget holders for the depreciation of capital items) can undermine the ability of SOF to hold equipment that is often obsolescent but may have utility in a certain scenario. SOF may be forced to shed assets that they would otherwise liked to have kept, but have been unable to accurately quantify to the satisfaction of the budget holders. ${ }^{19}$

- Culture - National culture has a major impact on the way SOF works. The controversy that followed Operation Flavius ${ }^{20}$ would have been a mystery in France or Israel as the SOF are, in many ways, expected to operate as they did. The American public is also rather suspicious of SOF operations, particularly in the Vietnam era with the MACV-SOG and the Phoenix programme. ${ }^{21}$ Such distrust can make the appropriation of much needed funds difficult. Culture also has an impact on the attitude to technology and the aversion to casualties. A society in which media focus can rapidly reduce political support will mean that the political authorities will try to avoid casualties, not only in one's own forces but those of civilians and enemy soldiers as well.

The third group of factors that affect SOF operations are internal, and are again categorised into political, economic and cultural:

- Cultural - The culture of any organisation can be characterised as its core paradigm derived from the interaction of several factors that shape the nature of the organisation. In the case of SOF, these can include the symbols or insignia of the organisation (in the case of the SAS, the winged dagger ${ }^{22}$ ), the use of a particular weapon (such as the M16A2 assault rifle instead of the SA80 in the 
case of the SAS), or the wearing of distinctive clothing (such as the SAS windproof smock or the Green Beret for the US Special Forces). The organisation itself is formally based on a traditional functional hierarchy similar to many organisations. SOF however, tend to have a slightly different dimension, which is based on the evolution of a process sometimes called the 'Chinese Parliament' 23 where individuals can question the execution of the mission regardless of their position in the hierarchy. This often manifests itself as a focus on the mission and is based on experience, often leading to an NCO dominance, rather than a functional focus, which is officer dominated. Too much of either will have profound effects on the SOF's performance. The final aspects of culture relate to the history of the organisation and the rituals and routines that build up over time.

- Politics and Economics - Politics and economics are bound up together and the internal organisational dynamics of SOF will be to do with the decision making process (who goes on what missions, who gets what equipment etc.). Decision-making can be classified into two areas, ${ }^{24}$ logical-empirical decisions (which are based on quantitative measures such as fiscal indicators) and normative-effective decisions (based on qualitative measures such as instincts, feelings and emotions). The decision to commit SOF to action are normally normative-effective, as they are based around the complex political dynamics that exist when that decision is taken. There is also the effect of personality, where the personality type most likely to be found in SOF is likely to be the nonrepressive type, who tend to make more emotive and intuitive decisions than the 
repressive type (who are emotionally fragile and thus less likely to be able to survive the long periods in a high-pressure environment).

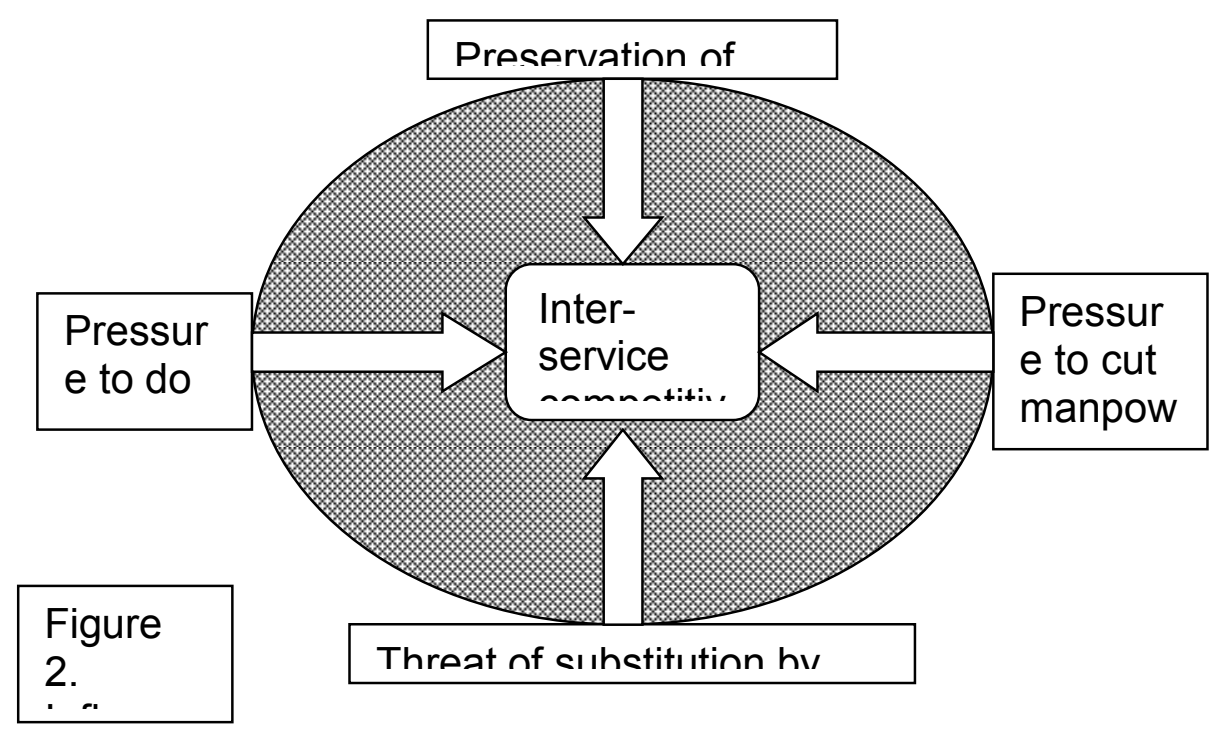

In summary, SOF are like most other organisations in that they are subject to forces both internal and external, and have to continually adapt to a changing environment, all of which have an impact on their logistic strategy as shown in the above diagram..$^{25}$

\section{SOF Logistics}

The concept of the supply chain has entered general usage in the commercial sector, and is steadily gaining credence in military circles, where it tends to be referred to as the support chain. It describes the co-ordinated flow of information and material between customers and suppliers from the emergence of a demand to the final delivery of the wanted item or service. In military terms it would cover the process from the initial procurement process to the final delivery to the front line 
user. $^{26}$ Logistic competence in the supply chain is derived through the successful co-ordination of network design, information handling, transportation, inventory and warehousing, material handling and packaging. ${ }^{27}$ Figure 3 below shows how the three key processes in the supply chain can be integrated through the flow of inventory and information, inventory in this case being the 'transformed input resource'28.

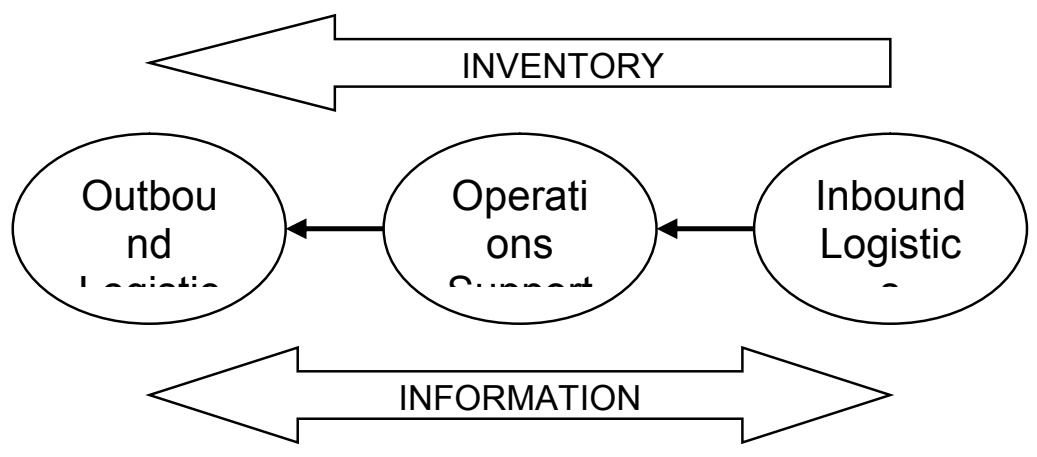

Figure 3. Generic

This model can be applied to most profit and non-profit organisations, and in this case, the support of SOF. Inbound logistics refers to the inputs with regard to suppliers and is concerned with the procurement of inventory (both capital equipment and consumables) as well as services to support operations, and could be stretched to include the recruitment, selection and training of personnel. The operations support area is concerned with those 'value-adding' activities that increase the utility of the inventory to the final customer (the deployed SOF). These can include modification of procured items or vehicles to suit operational conditions, or configuring items for airdrop. The outbound logistics function is concerned with the movement and receipt of items from the operations support area including the setting up of the distribution system if the SOF are deployed. 
A generic SOF supply chain (based on the previous diagram) is shown in Figure 4:

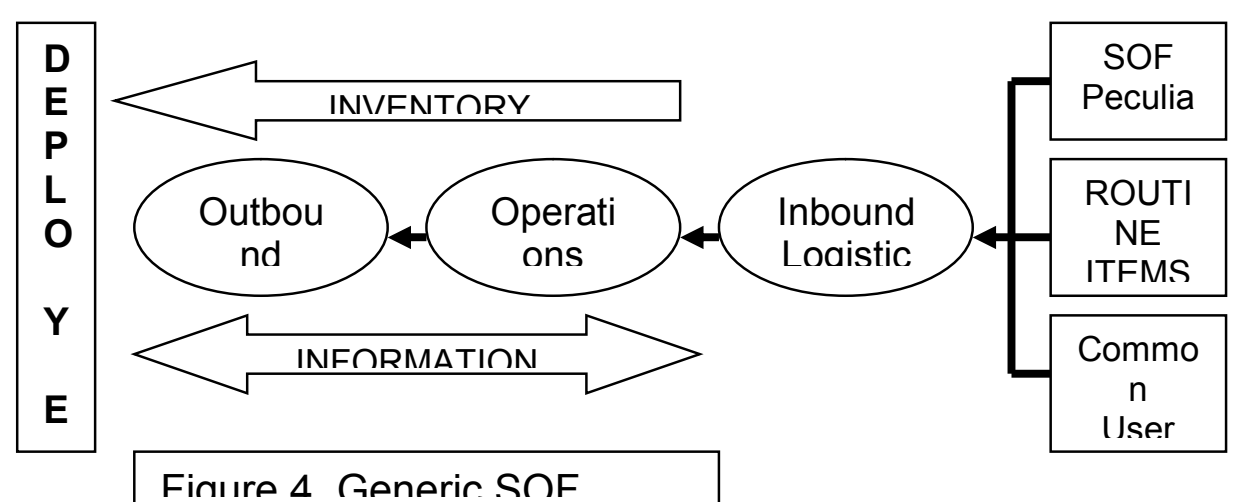

Finu ure $\Delta$ Generir SOF

The SOF distribution system is a network running from a Point of Embarkation (POE), usually an airhead (APOE), through an Intermediate Staging Base (ISB) ${ }^{29}$, often outside the area of operations or even afloat ${ }^{30}$, through nodes to the deployed SOF. SOF resupply and medical evacuation operations differ from conventional forces as these are planned and executed as operational missions, as opposed to being treated as logistic responsibilities. From the ISB, a Main Operating Base (MOB) may be deployed, depending upon the size of the operation and the geography of the area. If not, then the chain would go directly to a Forward Operating Base $(F O B)$ that would be sited quite near to the area of operations but never in enemy territory. From the FOB, the SOF will deploy to the operational area or perhaps an Advanced Operating Base (AOB). They will be resupplied by land, through sea Distribution Points (DP) or Helicopter Landing Sites (HLS). Beyond the FOB, logistic operations become an integral part of the combat operation. 


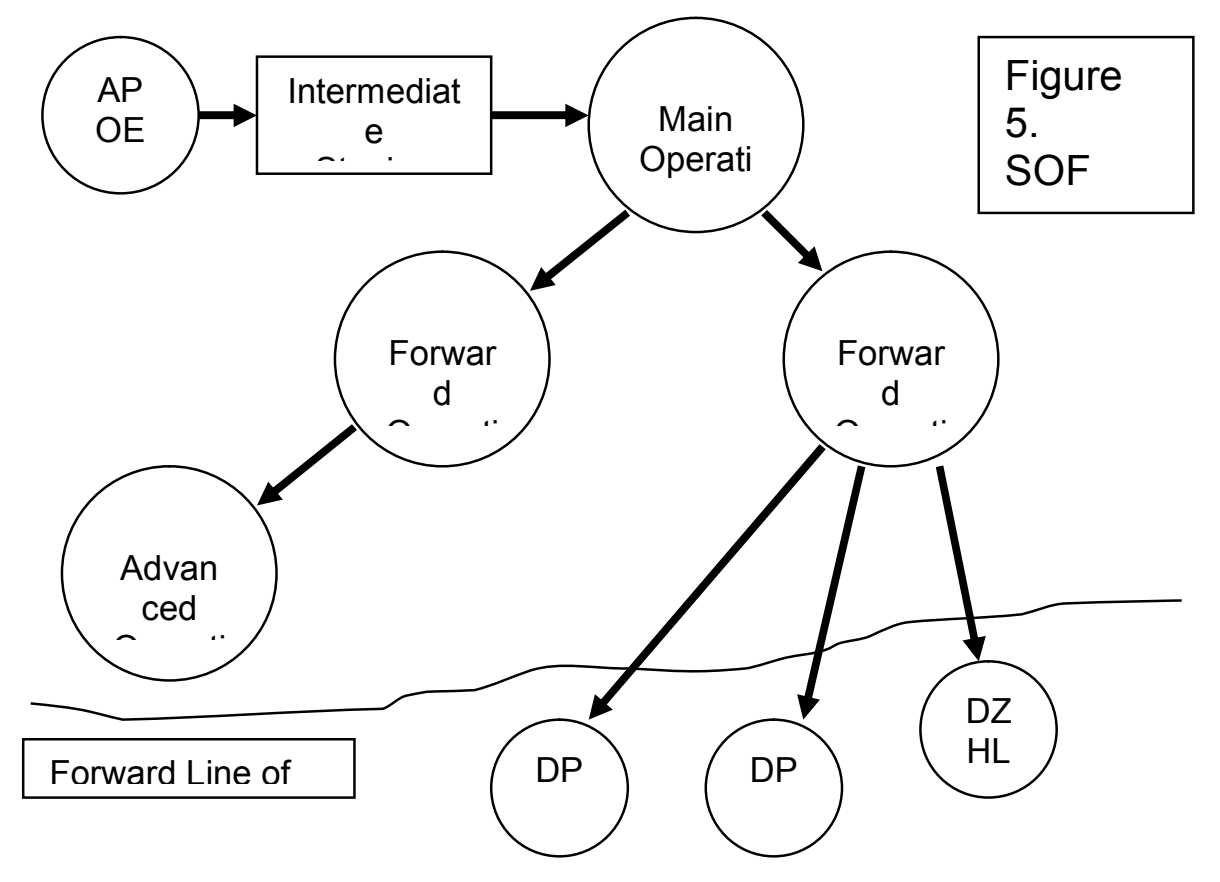

Information flows within the SOF supply chain link the logistics activities together into an integrated whole. They contribute to the development of the most effective logistic strategy to support deployed SOF whilst balancing the influences previously mentioned. This integrated process builds on four levels of functionality: ${ }^{31}$

- Transactional - concerned with the basic initiation and recording of logistic activities such receipts and issues.

- Management Control - concerned with the performance and reporting of the system and uses measures such as financial cost, turn around time and quality measurement.

- Decision Analysis - the level at which alternatives are considered depending on the information coming from the lower two levels. It is geared towards process improvement. 
- Strategic Planning - provides support to the development and refinement of logistics strategy.

Inventory is input into the supply chain in order to reach the SOF, whether deployed or not. SOF may source particular items through dedicated research and development via funding from their own budget, or acquire items in common use throughout the armed forces for reasons of commonality, economics, and simplicity. Many of these items are often allocated centrally with the SOF being just another user. Other items may be commercial-off-the-shelf (COTS) goods that are sourced to meet a specific purpose, outside of the normal procurement procedures. The items that are acquired, and are to be used in operations, will need to have a high degree of reliability, be simple, inter-operable and be easy to maintain, as SOF often operate in austere conditions and the high operational reliability requirement may necessitate a larger than normal spare parts inventory.

The distribution (transport) system is the key to the SOF operations and is where logistic support faces a stern challenge. It is not merely a support function but very much a core aspect of special operations. A resupply mission is likely to have to cross deep into enemy territory in a clandestine manner ${ }^{32}$ and is therefore in just as much danger as the deployed unit. SOF will use any transport that they deem appropriate, which could include fixed wing aircraft (such as the MC-130 Combat Talon), rotary wing aircraft (such as the MH-53 Pave Low, CH-47 Chinook or MV-22 Osprey), fast jets dropping high speed containers (such as the A-21), land vehicles (such as the UK's Medium Support Vehicle (MSV)) and water transport (such as the Rigid Inflatable Boat (RIB) and submarines). 


\section{SOF Logistic Requirements}

The paper has looked at the factors influencing SOF operations and the structure of the supply chains. It will now consider the logistics requirements it must meet in order to be successful.

The logistic support imperatives for a SOF logistic system can be distilled into six key areas:

- Self-sufficiency - it is of fundamental importance for the SOF logistic system to allow the operation to be as self-sufficient as possible, and minimise the number of resupply activities that may be needed.

- Demand pull - the nature of SOF missions means that traditional automatic resupply (push) logistics is inappropriate due to the high-risk operations that SOF undertake and just to mount a resupply mission deep into enemy territory on the off-chance that the SOF may require resupply is unacceptable. SOF logistics must therefore deliver what is required, when it is required and where it is required. Commercially, this is known as Just-in-Time logistics. The only difficulty in implementing this is that communications must be as near to 100 percent reliable as is possible and there must be a contingency plan in operation in the event there is a communications failure.

- Operational security (OPSEC) $)^{33}$ - this is a key factor, particularly so given that the changing nature of warfare means that the exact character and identity of the enemy may (initially) be unclear. Any logistics strategy and structure must take this into account and cannot compromise it. This means that sourcing must be 
carried out with care and vendors should be closely examined so that mission security is not breached. Careless sourcing has the potential to endanger the lives of those personnel carrying out the mission (as it may with any military operation) and so the exact location of the operating bases should be concealed when making delivery, even in a friendly and benign environment, somewhat problematic.

- Freedom of action - as logistic drag can severely affect Special Operations, logistic requirements must provide the minimum constraints upon an operation and so the logistic system should avoid the over-insurance that occurs in a 'Just In Case' inventory system. This is particularly so for those operations which require agility. This has to be balanced against the need for self-sufficiency to avoid over-loading men ${ }^{34}$ and vehicles ${ }^{35}$. This need to minimise logistic drag has to be traded off against the need not to restrict options due to the unavailability of supplies or equipment ${ }^{36}$. It is a difficult balance to achieve and a stern test of a logistician.

- Interoperability - with regard to SOF, interoperability (the ability of systems, units and forces to operate together in order to accomplish the given objective) operates on two levels. The first is the national level; Special Operations rarely happen in isolation (although that may change) and usually occur as part of a larger Joint Force and to minimise delay, the SOF will use the existing in-theatre logistic system. This can be made harder when equipment is not completely interoperable. For example, some European SOF prefer to use the American M16A2 assault rifle, which cannot fire the UK manufactured SS109 $5.56 \mathrm{~mm}$ round as effectively as the US manufactured M193 $5.56 \mathrm{~mm}$ round. Conversely, some European small arms, for example, the British SA80 assault rifle, cannot 
fire the M193 as effectively, although the US M16A2s have been upgraded so that they can fire either the SS109 or M193 round equally well. Therefore UK SOF have to bring in additional stocks of US manufactured $5.56 \mathrm{~mm}$ ammunition, rather than draw on ammunition supplies from other UK forces. ${ }^{37}$ As another example, the US Air Force MH-53J Pave Low helicopters (used to support Special Operations) have become so specialised with various upgrades that they have only a limited commonality with other US Air Force $\mathrm{HH}-53$ or US Marine Corps $\mathrm{CH}-53$ helicopters, which were all based on the same airframe. The second is the international level. The changing nature of war and how it is to be fought means that Joint Special Operations Task Forces (JSOTF) are more likely to be required to operate together, although some 'de facto' interoperability exists as many alliance partners have to buy US equipment as they do not have the resources to develop indigenous equipment themselves. This has already occurred with the US PRC-137 HF radio being in widespread use across NATO.

- Accountability - with regard to SOF, accountability is in both financial and political terms. Logistic systems are used to create a proper audit trail to try and minimise waste and maximise value for money. The UK has set up the Defence Logistics Organisation (DLO) and introduced Resource Accounting and Budgeting (RAB), putting a greater emphasis on 'lean logistics' and a reduction in the amount of stores and supplies held in inventories. Political accountability is just as important however, with SOF operations receiving a great deal of attention and sometimes becoming involved in legal proceedings. The logistic system should maintain visibility of assets not only during the operation but for sometime afterwards as well. 


\section{Meeting the Challenge of SOF Logistics}

Having looked at the factors influencing SOF operations, how their supply chains are made up and the requirements it must meet in order to be successful, the paper will now put forward an approach (and in doing so develop a hypothesis) to solving the problems of logistics support to SOF, utilising case study examples of SOF activities and logistic support.

The risk of failure in an operation increases according to the scale of the operation, the nature of the role the SOF are undertaking and the type of SOF being used. There are three operational scales, Task Unit (individual elements or teams, vehicles or aircraft - unlikely to be used alone), Task Group (made up from Task Units and is self-sufficient in terms of insertion and extraction assets) and Task Force (made up from Task Groups). The first stage in putting together the approach, involves constructing a hypothesis. Firstly, we will assume that the level of risk to an operation from logistic failure increases as the scale of the operation increases (see Figure 6). 


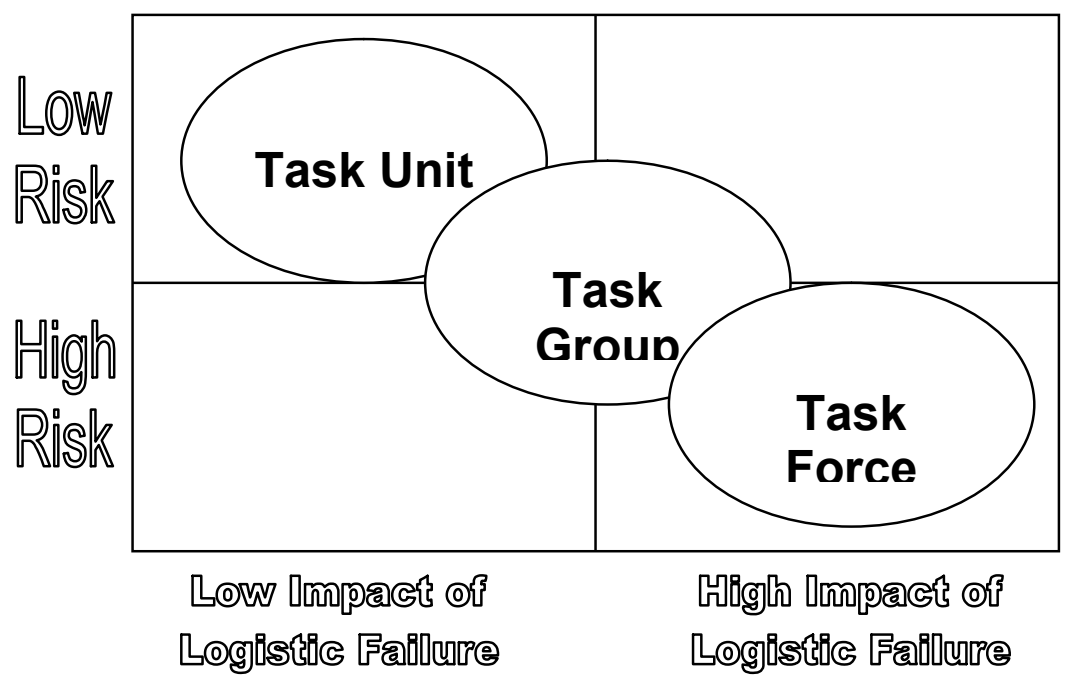

Figure 6. Logistic Implications of Increasing

The nature of the role was highlighted earlier, and was split into 'traditional', 'low intensity' or 'operations other than war' and 'peace operations'. The second part of the hypothesis stipulates that the level of risk associated from logistic failure increases as the nature of the role changes (see Figure 7).

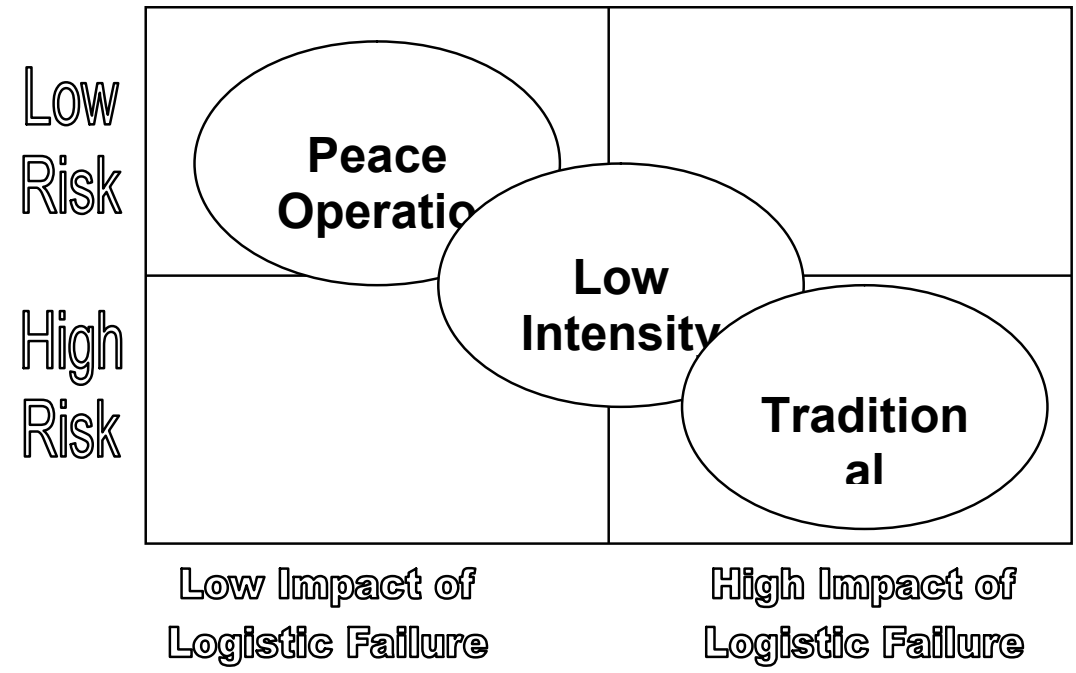

Fiqure 7. Loaistic Imnlications of 
Thirdly, as SOF generally fit into three models (as highlighted earlier) the hypothesis will stipulate that depending on what model the SOF fits, this will have an impact on the level of risk associated with logistic failure (see Figure 8).

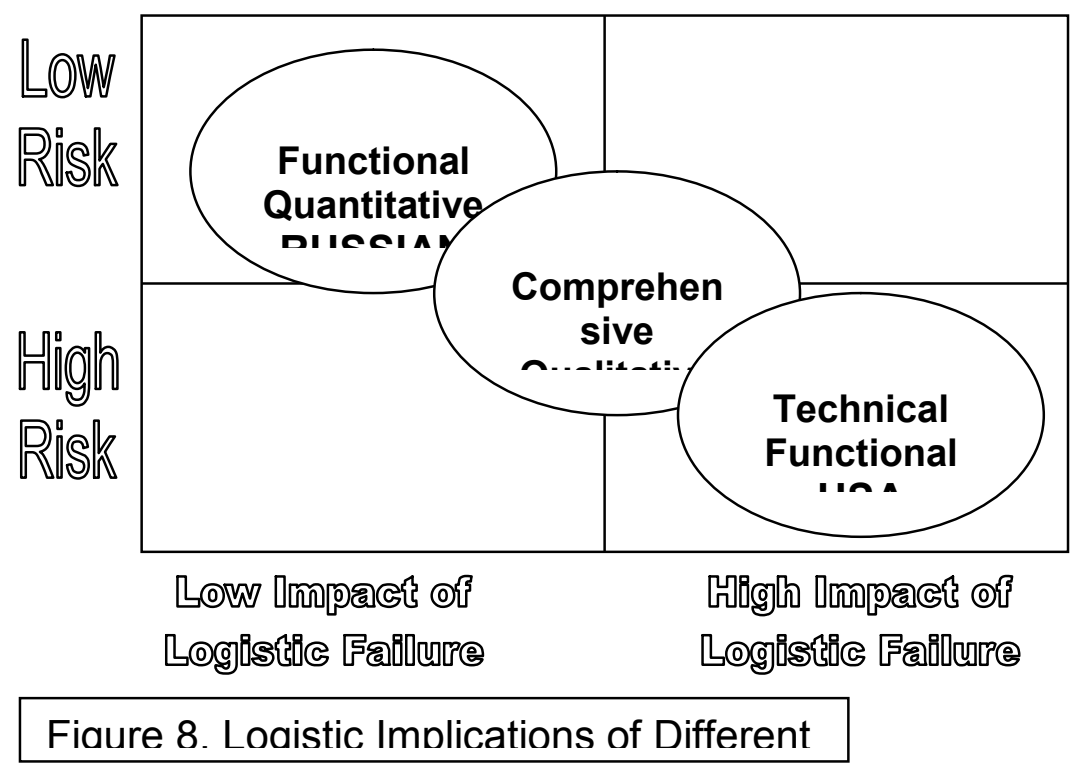

These three aspects can then be combined into a 3D-cube model, which could provide a means to differentiate the different logistics strategies that would be appropriate for any operation based on scale, role and model of SOF. From that, a specific logistics strategy for a given operation could be derived using Best Commercial Practice to ensure efficient and effective support. 


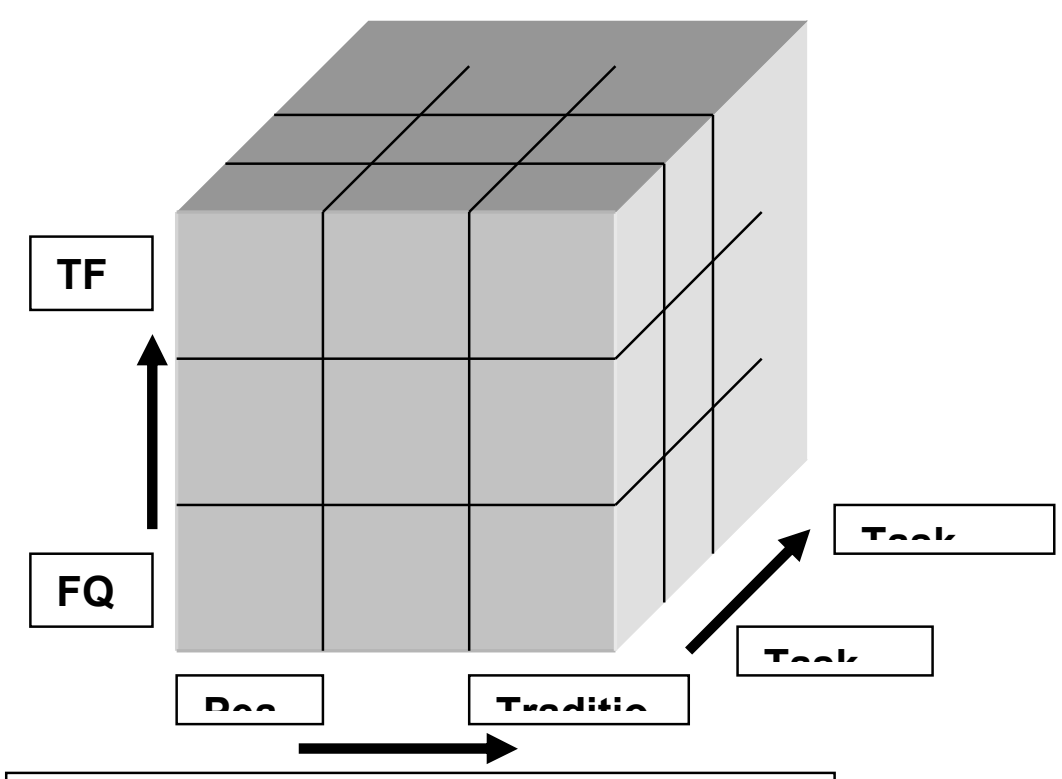

Figure 9. Combination of Risk Impact

Mntrinan in CNC Cunnart M MaNal

This 3D model could be read as:

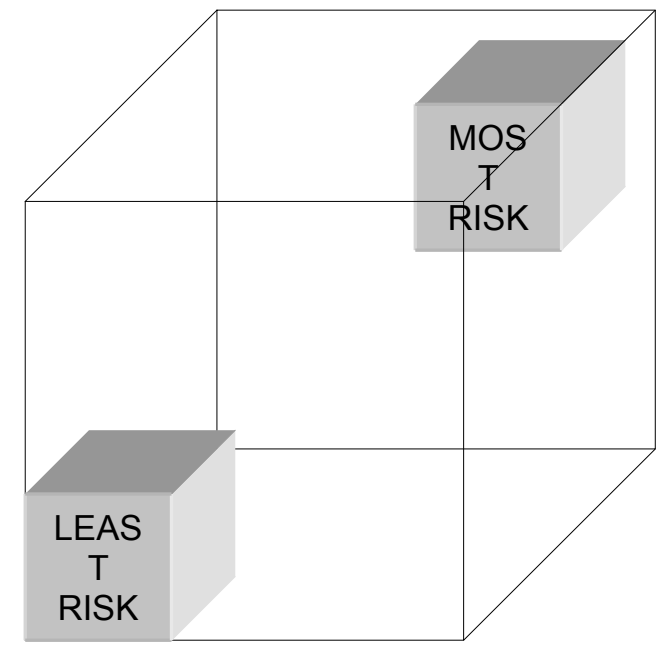

Figure 10. Interpretation of 3D SOF 
The testing of the assumption that lies behind the hypothesis involved the selection of some thirty-two case studies that were drawn from a wide variety of historical contexts in terms of model, scale and role. The cases were selected on the basis that:

- They represented an extraordinary logistic challenge, such as the Israeli raid on Entebbe in 1976, codenamed Operation Jonathan.

- They provided a powerful contribution to a larger operation, such as Operation Prelim, the UK SOF attack on Pebble Island during the Falklands Conflict of 1982.

- They proved to be a major operation in their own right, such as the attempt to extract US PoWs from Son Tay in North Vietnam in 1970, codenamed Operation Kingpin.

- They were representative of a particular role, such as the rescue of BAT21 (as dramatised in the film starring Gene Hackman) in Vietnam in 1972.

- They were representative of the SOF model, such as the Spetsnaz operations in Levant-Catalonia during the Spanish Civil War in 1937.

- They were of a particular strategic significance, such as the rescue of Benito Mussolini by Otto Skorzeny and his Commandos from Gran Sasso in 1943.

Each case study was examined in order to produce an assessment of the logistic risk taking and logistic impact of mission decisions. Each case study was then examined against two sets of criteria, the first being what the likelihood was of a failure in the support chain and the second was what the impact was (or would have been) of a failure of the support chain. Such factors that were considered in the case of the impact of failure were the degree of specialisation, mission key 
equipment, the inability to adapt to logistical failure, system redundancy and back up, and spare resources. With regard to risk minimisation, those factors that could mitigate the risk of logistic failure included ability to repair, logistic planning, support chain analysis, ability to resupply, the overall importance of personnel as regards the ability to evacuate casualties and with reference to the previous category, the provision of medical support. These factors had been based on an initial examination of a number of case studies where logistics had clearly proved to be a deciding factor in one of two ways. Firstly, where logistics clearly had a contribution to the success or failure of the mission, and secondly, how well the logistic support was carried out. Each factor was given a numerical value, and the mean taken, resulting in two values for each study. In terms of the impact of logistic failure, a high value would indicate that it had a seriously detrimental affect on the operation, while for the likelihood of failure, a low value would mean that the risks had not been sufficiently minimised through forward planning, establishment of the proper logistic capability etc. These values were then plotted on a graph, the $\mathrm{x}$-axis representing logistic impact, while the y-axis represented risk minimisation. 


\section{Figure 11. Chart of Case Study Data Results}

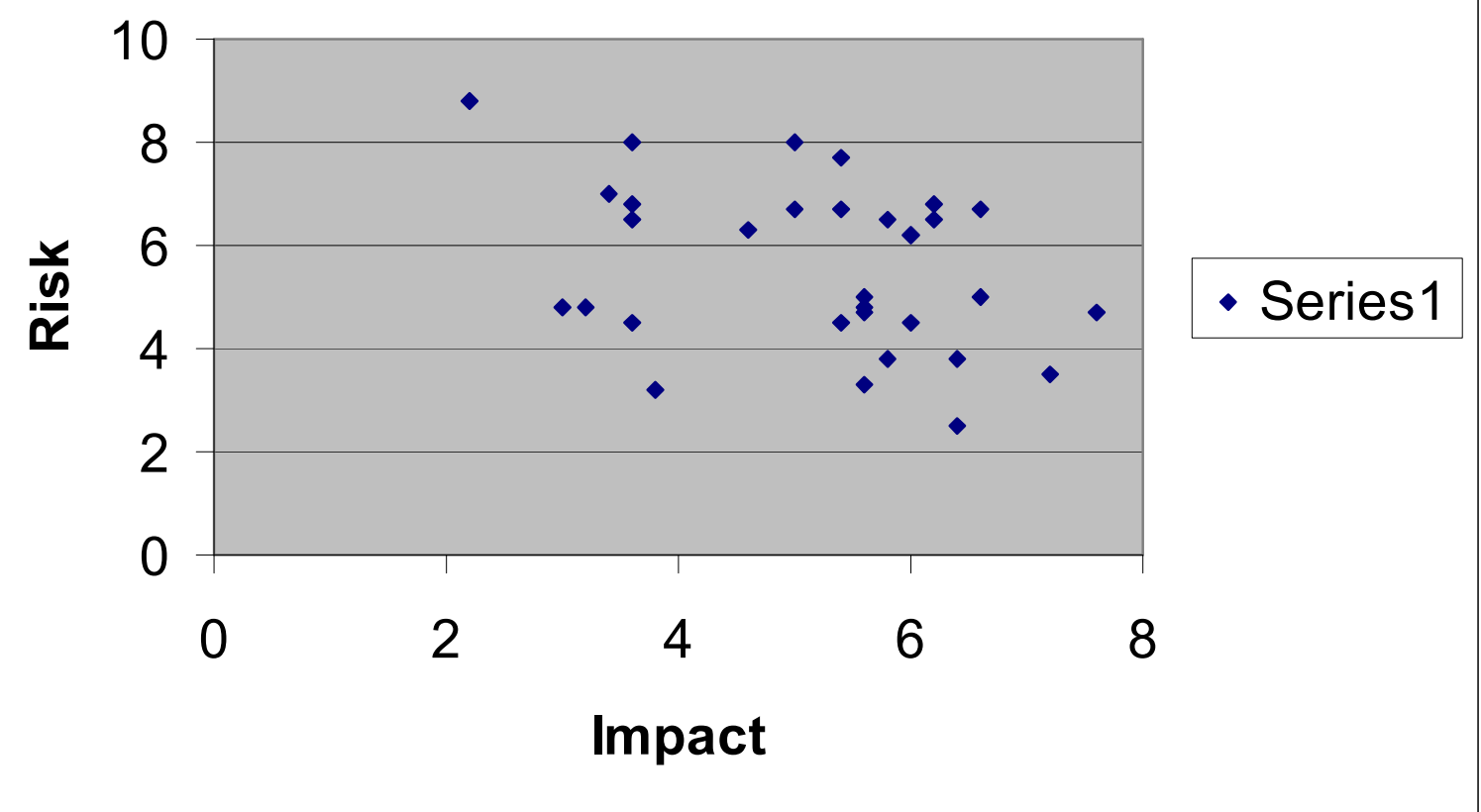

The next stage consisted of interpreting the results with regard to the three main determinants, that of scale, role and model.

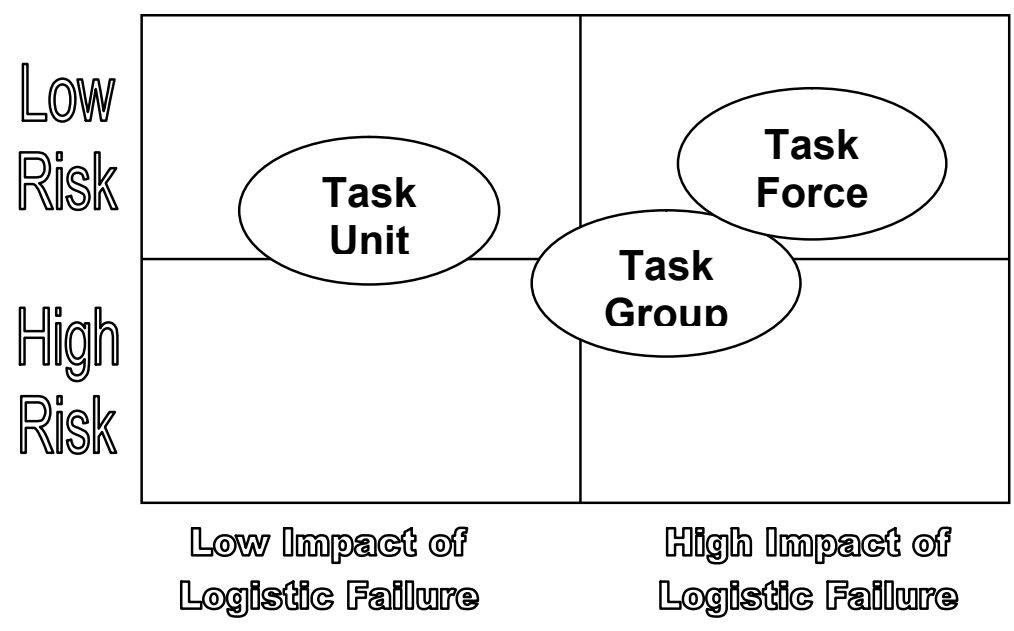

Fiqure 12. Case Studv Results 


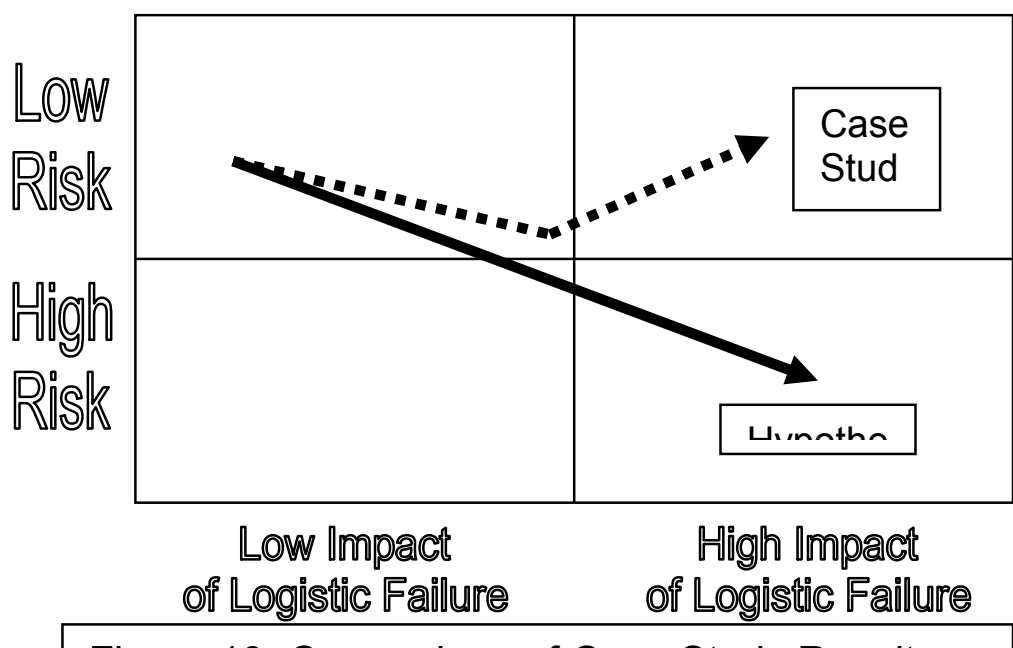

Figure 13. Comparison of Case Study Results

The original hypothesis put the Task Unit (the lowest level of operation) in the low risk / low impact quadrant because it was thought that lower force levels would create fewer logistic problems and as it would be possible to achieve a higher degree of self-sufficiency, a failure in the logistic chain would have a lower impact. While this seems reasonable given the data, the rest of that part of the hypothesis, i.e. that as the level of the operation increased so did the risk and impact of logistic support chain failure, was not quite so clear. Task Group operations seem to confirm this but the Task Force operations seem to be at a lower risk of failure than anticipated. It is probable that only those countries (such as the USA and Russia) that have a large, well-developed capability can mount operations such as these and that they have adequate resources to properly support, and therefore minimise, the risk of logistic support failure. 


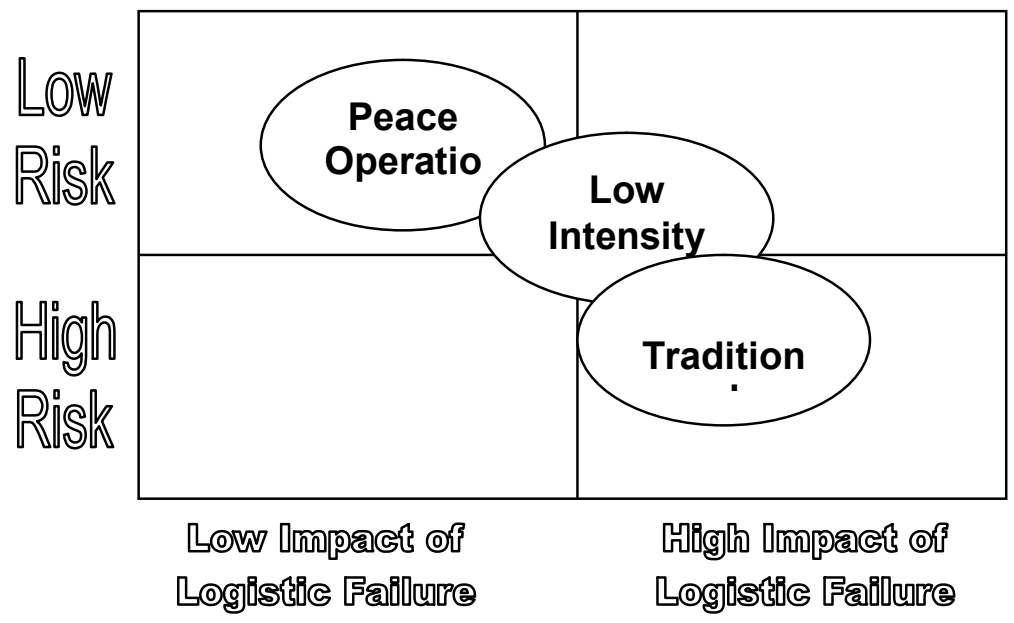

Fiqure 13. Case Studv Results

The hypothesis suggested that as operations moved away from peace through ever more intensive (and dangerous) operational situations that the risk and impact of logistic failure would increase. Analysis of the case studies seems to bear out these prepositions.

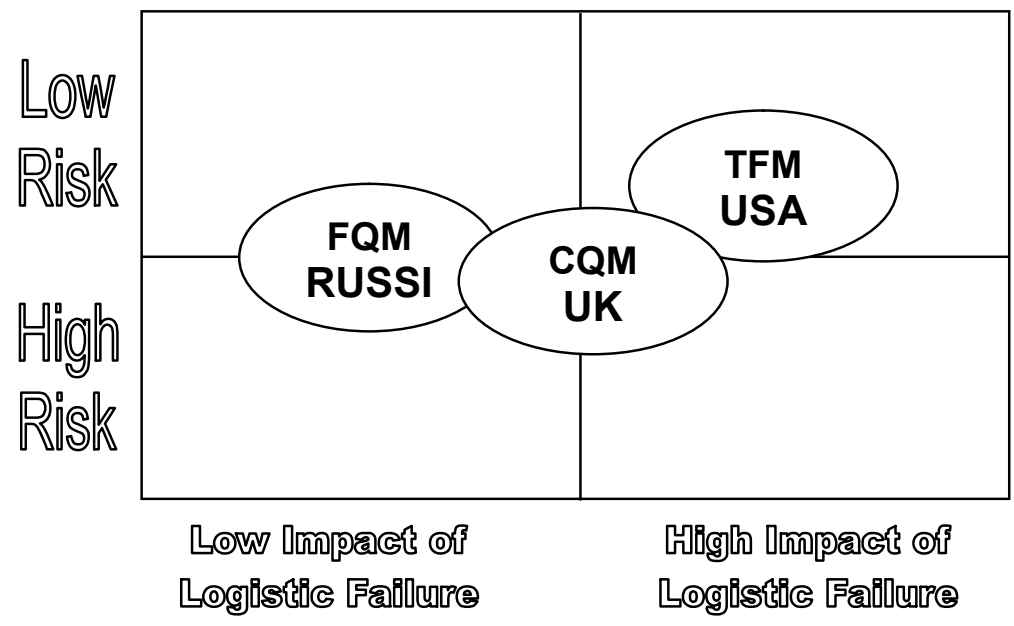

Fiqure 14. Case Studv Results 


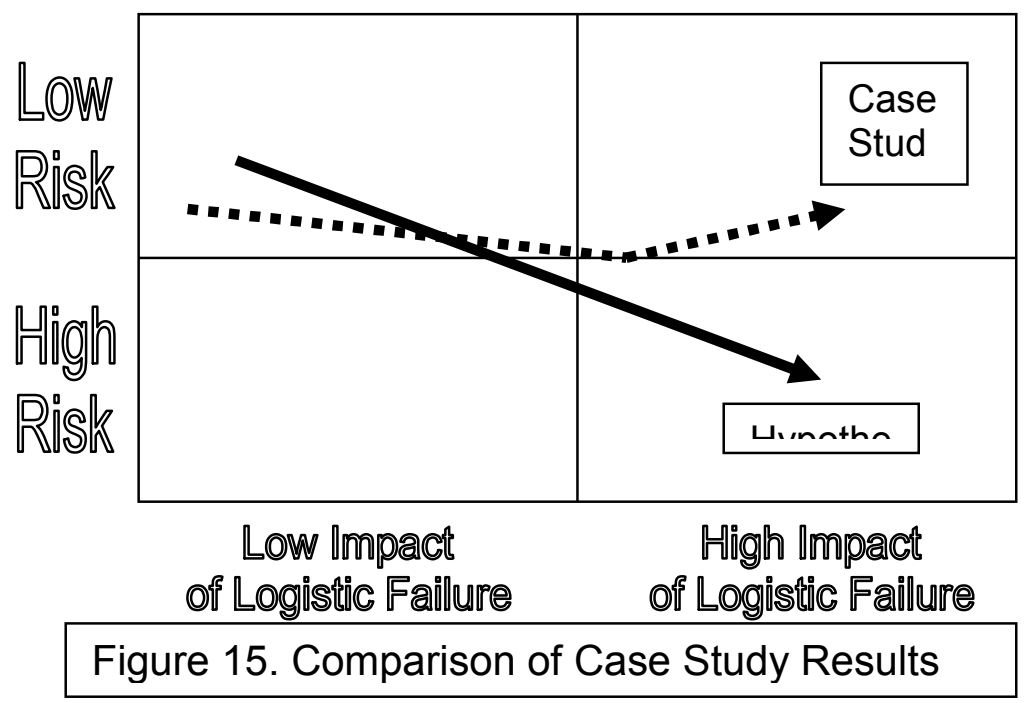

The hypothesis stated that the Functional Quantitative (FQM) or Russian model would be low risk and low impact as there is a lower level of technology generally and that standard issue equipment tends to be used which limits the logistic burden. FQM operations rarely operate above the Task Group level ${ }^{38}$ and FQM SOF have a limited range of roles compared to other SOF and so can standardise logistic procedures more easily. The Technical Functional (TFM) or US model was felt to be at the high risk / high impact quadrant as the use of high technology generates equipment support issues and the wider variety of roles means a wide variety of equipment which increases the logistic burden. The operations tend to be larger with a wider range of specialised equipment and the increased reliance on technology will increase the impact of logistic failure. The Comprehensive Qualitative (CQM) or UK model was thought to sit between the two extremes.

The case studies did support the general ordering of the models and the data is consistent with the hypothesis in terms of the impact of logistic failure. However, the 
data differed in the degree of risk minimisation within the models. There is a slightly higher degree of risk in relation to the FQM model and somewhat lower in relation to the TFM, so while the impact of logistic failure is still markedly different, the risks involved are broadly similar (although the TFM is slightly lower). This trend appears to originate in firstly, the increased emphasis in force protection ${ }^{39}$ in Western Armed Forces that may mean that the most complex and risky operations are not carried out at all. Secondly, the aversion to casualties means that TFM operations will involve extended planning and over-insurance with regard to logistics and so be very slow to respond to contingencies. ${ }^{40}$ Thirdly, the FQM may be distorted by operations such as Chechnya where the Spetsnaz have been used as little more than elite infantry in inappropriate roles. The poor state of Russian logistics is unlikely to help matters.

Overall, most of the tenets of the hypothesis were upheld, with the data being inconclusive as regards the degree of risk minimisation in relation to the model of SOF employed. In summary: 


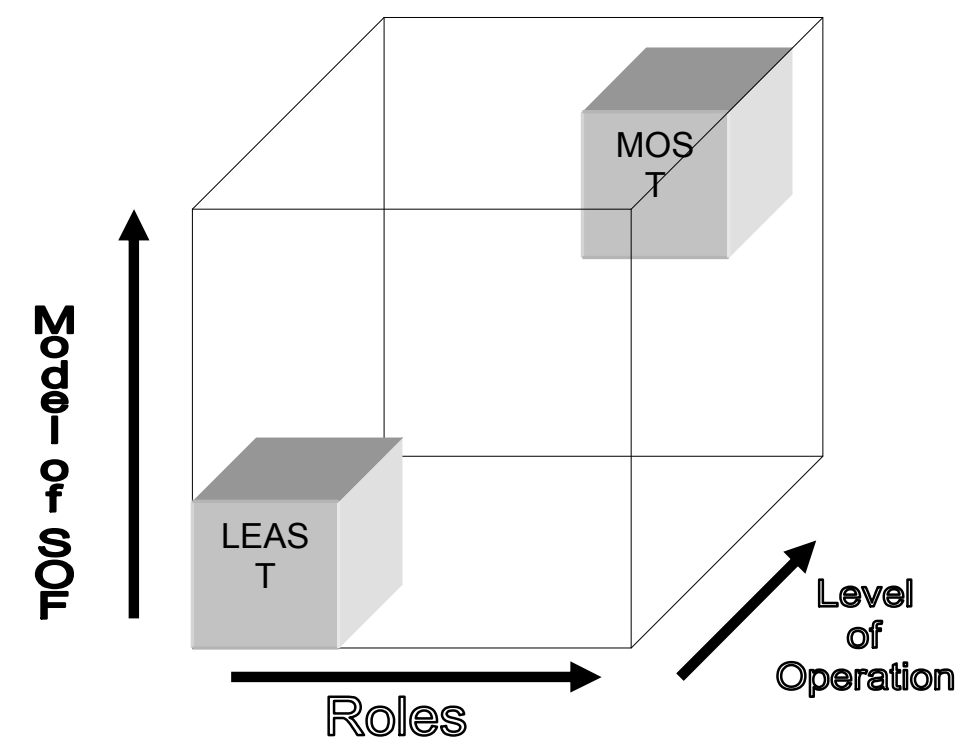

Figure 16. Combination of impact matrices on SOF

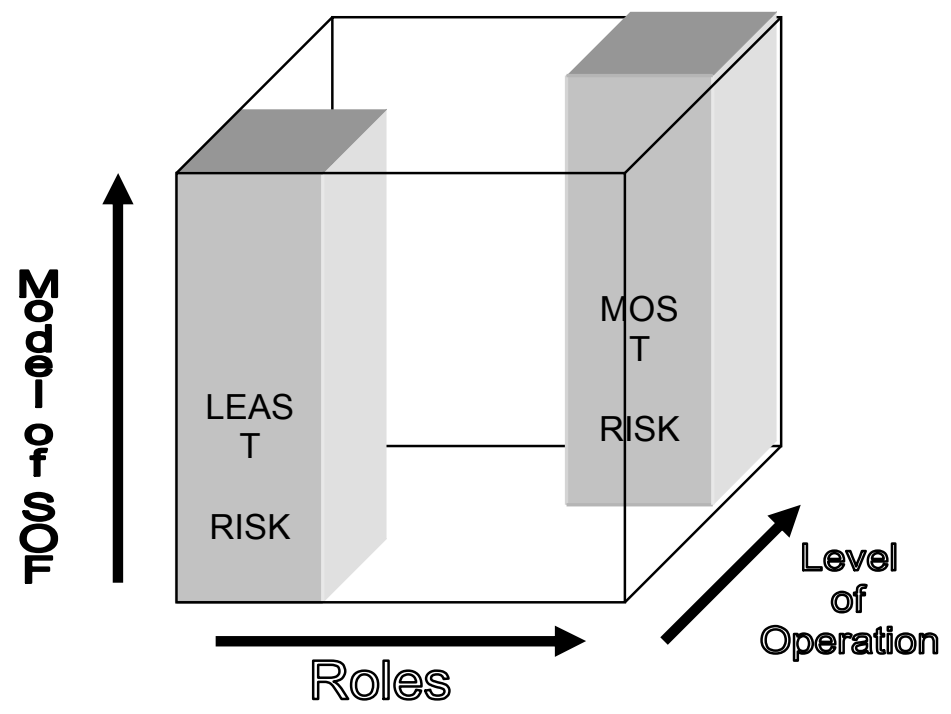

Figure 17. Combination of risk matrices on SOF 


\section{Deciding the Allocation of Resources for SOF Operations}

Having analysed the validity of the hypothesis through the application of data derived from case studies, how can this be applied to the problem of logistics support to SOF? Looking back, the paper has previously referred to the necessity of a logistic system being able to respond to these imperatives:

- Self-sufficiency - wherever possible, SOF should be self-sufficient in order to reduce the need for resupply and reduce the possibility of the mission being compromised. There is of course a finite weight that a soldier can carry (even a member of the SOF) without reducing performance. ${ }^{41}$ Reducing weight requires investment in lighter weight alternatives that are usually more expensive than their normal counterparts as they often require research into new materials. This obviously will have an impact on the budget allocated to the SOF. There may be fewer opportunities to reduce the unit cost as the high initial price will make the item less attractive to the rest of the armed forces (and even the civilian market if applicable) and therefore the supply of such equipment will be less attractive to potential manufacturers. It is therefore unlikely that buyers of this equipment will be able to benefit from economies of scale.

- Demand Pull - in order to reduce the inventory held by a deployed SOF that has a finite capacity to carry what it needs with it (and therefore achieve selfsufficiency) the philosophy of Just-in-Time (JIT) could be applied to the logistics support of SOF. Items would only be delivered as and when they are needed. This has a number of implications for logistic support. There must be sufficient depth and breadth of items held within the support chain to meet SOF requirements, and these will necessarily have a short lead-time. There must be 
a secure method of communicating the requirement up the supply chain and the distribution system must be able to deliver items into what may be hostile territory without compromising the operation. This has a significant impact upon the inventory, storage and distribution requirements of the logistic system. Such a system will need investment in order to guarantee that it has the requisite responsiveness and inventory does, after all, represent money that is tied up. Storage requires investment in facilities that will hold items and reduce the chances of that item deteriorating whilst the distribution system will need investment to achieve the capability of penetrating enemy territory undetected. ${ }^{42}$

- Operational Security (OPSEC) - this is paramount to special operations and any logistics system must take account of this requirement. This has implications in that firstly, items may have to held a long time in storage as sourcing on an 'as required' basis may breach OPSEC and compromise the operation. ${ }^{43}$ Secondly, visibility of inventory can be a problem in conditions of high OPSEC, so safety stock may have to be held to take account of instances where deliveries cannot get through. ${ }^{44}$

- Freedom of Action - this refers to both giving the commander flexibility through having the necessary inventory available as well as not constraining them through logistic drag. This can often be a difficult balance to achieve.

- Interoperability - being able to operate and be logistically compatible with other nations as well as the rest of the country's armed forces can help the logistician through economies of scale and availability of spare parts, consumables and equipment. They can however, be difficult to achieve simultaneously to any great degree, as there is always going to be an element of differentiation in national procurement for geographic, strategic and political reasons. There has been a 
degree of standardisation in NATO SOF around the use of the M16A2 and the Heckler \& Koch MP5. These items are not standard issue within the UK military however, and so create a problem when the UK SOF are deployed with the remainder of the armed forces.

- Accountability - this is a problem facing Western SOF more than other nations in both political and financial terms. Political accountability is concerned with the legality of certain operations and the logistic system is closely tied up with the preservation of evidence and the visibility of equipment (to make sure that has been used correctly and achieving value for money if nothing else). There is pressure for this not only on operational deployments but during peacetime training as well, where accident inquiries can require the same degree of accountability as found on operations. The pressure since the end of the Cold War to reduce defence budgets has meant that SOF are even more accountable for their expenditure and it often falls to the logistic system to control costs.

It is now pertinent to examine how the hypothesis outlined earlier, can help in resolving these often competing demands. Resources are finite for the armed forces as a whole, and SOF are seen by many parties as a luxury item and so SOF must be seen to make the most effective use of the resources given to them if they are to receive continued political and financial commitment. All logistic systems have the objective of providing all their customers with the level of service they require but finite budgets mean that priorities have to be set. ${ }^{45}$ In commercial terms, this means that recognition is given to the fact that not all customers are equally profitable and so the highest service is given to those key customers and key products. ${ }^{46}$ However, 
the profit motive is difficult and inappropriate to translate to the allocation of resources to SOF.

It should therefore be possible to differentiate on the basis of the factors used earlier in the hypothesis. Resources could be allocated against the factors of risk and impact, rather than profit and volume. The SOF support model can be used to decide upon the allocation of resources based on the logistic analysis of the nature of the operation, in particular the role and scale. The aim of this would be to derive a solution to the conflicting demands upon the SOF and decide how best practice can be used most effectively to improve the allocation of resources.

\section{Logistic Strategies: Balancing Risk and Impact}

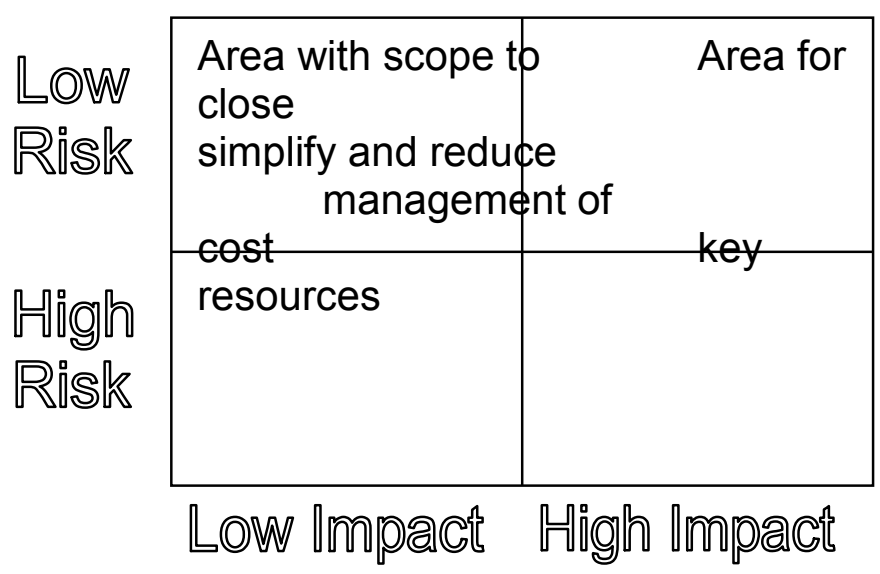

Figure 18. Matrix showing risk and impact

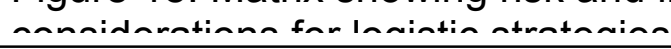




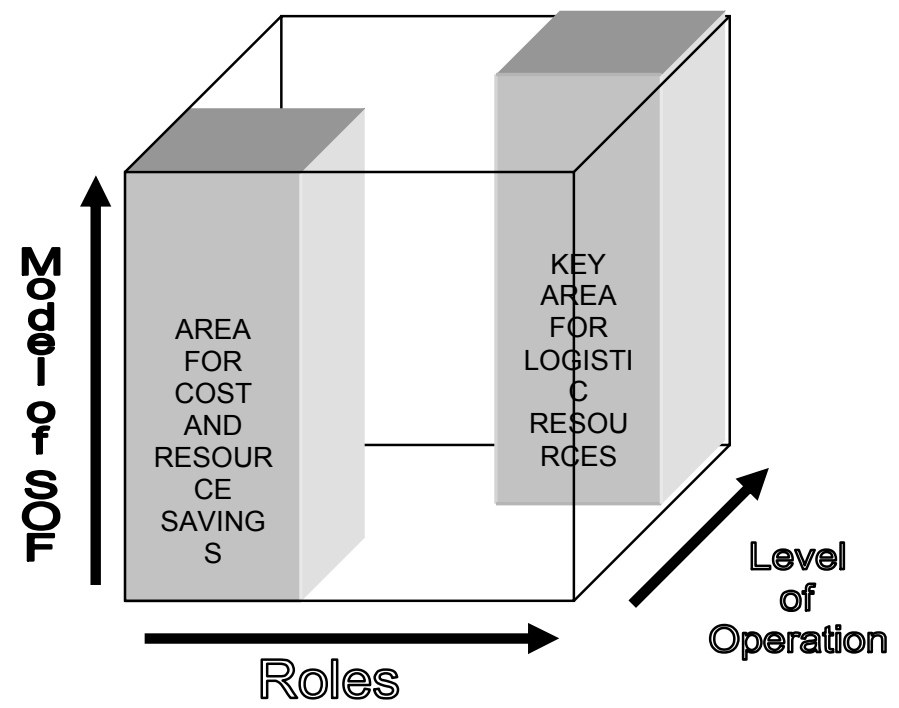

Figure 19. SOF Support Model used to differentiate

The simple matrix shown suggests how the balance between risk and impact can be used to suggest alternate logistic strategies. It could be summarised in a three dimensional model shown above.

Having looked at the possibility of differentiated strategies, the paper will now analyse how these strategies could be implemented and what commercial 'best practice' can offer in improving effectiveness. It will now examine each broad area in turn and discuss how a logistic strategy could develop.

The area of high-risk, high impact operations (where there is a high risk of failure and a high degree of impact following such a failure) is what can be considered the core competence of SOF. Activities in this area are crucial to the execution of a mission and are by definition the most difficult to carry out. The SOF support model 
suggests that it is the traditional activities carried out at the Task Group level or higher by Western model SOF that fall within this area. The SOF logistic imperatives are of key importance here and logistic resources must be concentrated accordingly. As this area is a core competence for SOF, the application of commercial best practice would suggest that outsourcing ${ }^{47}$ these activities would not be beneficial for the following reasons:

- Loss of control over support activity

- Potential OPSEC breach

- Lack of visibility

- Reduction in flexibility

- Operational risk

If one considers that this area above all others is at the core of the SOF's business, then what should the strategy encompass? The key elements to any strategy are information, inventory, storage and transport and distribution.

- Information - information flows are the links that turn logistic activities into an integrated process and work at all levels from the simple transactional level to the strategic planning level. Any strategy in the high-risk, high-impact area should address firstly, that communications are vital to all levels of an operation. At the transactional level, communications enable the effective execution of transactional logistics and it is vital to the operation of the logistic imperatives, in particular the need for demand pull. Communications systems must have an element of redundancy, in addition to procedures in place in case the systems fail. Secondly, visibility ${ }^{48}$ is vital to allow effective use of scarce resources and to 
allow redirection where necessary. ${ }^{49}$ This visibility needs to take account of the concerns of OPSEC and be consistent with the desire for self-sufficiency. Thirdly, planning input is vital. Resupply of SOF is not a routine administrative activity but a mission in itself. Logistic information input is vital to ensure that the minimum number of missions are carried out to reduce the potential for breaches of OPSEC. The key to logistics planning is the formulation of contingency plans to cover such situations as lost communications.

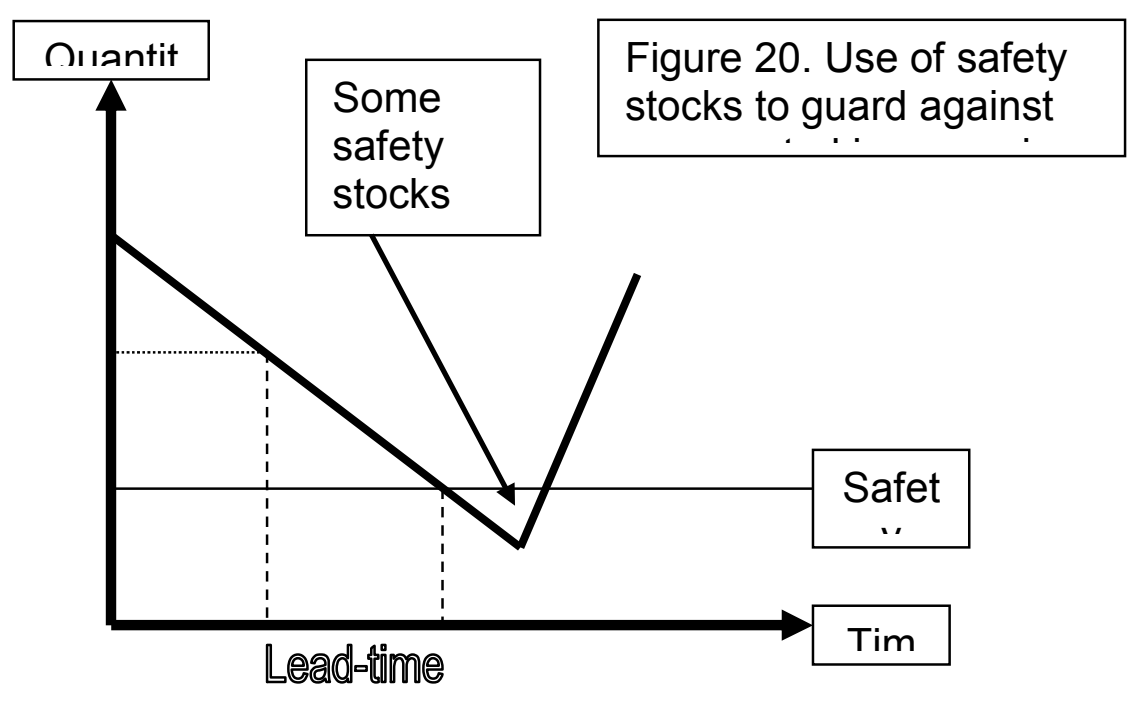

- Inventory - as the transformed input resource inventory represents the 'fuel' of the SOF engine and the key capital cost items of any operation, the central imperative in this case is self-sufficiency. The sourcing of items within an appropriate lead-time must be balanced against the desire to be completely selfsufficient. This balance needs to ensure that there is a mission duration stock of SOF particular items that may be held at various points in the supply chain. These items should be held in an allocation consistent with the ability of the Task Group or Force to transport it within an acceptable time. Alongside this, there is 
a requirement for the visibility of common user items that might be held in the system and the capability to divert part of those stocks to SOF. Central to this would be the maintenance of a safety stock to account for differences in time between demand and supply. Routine items, which are sometimes termed MRO (maintenance, repair and operating) items, are not as capital intensive and have minimal OPSEC implications. These items can be sourced widely with some safety stock consistent with local availability. In times of poor local availability, these stocks will be of a higher level with storage and accountability implications.

- Storage - the storage of inventory items can be of considerable importance. The larger communication requirement means that SOF support locations generate a considerable amount of transmission radiation, which is sometimes inconsistent with the storage of key inventory items such as guided missiles, which require Radio Frequency (RF) overpacks to shield key electronic components. As the amount of these communications increases so does the requirement for shielding. Storage areas must be sufficiently close to meet the lead-time requirement and be secure. SOF peculiar items also need considerable security, not only because it can indicate their presence, but also because these items are often nearly impossible to replace in a short space of time.

- Transport and Distribution - the ability to move inventory is vital to the operation of the SOF logistics system and its capability dictates where and how a large proportion of the inventory can be held. The difficulty in forward storage, and often limited transport capacity, dictates that the further up the supply chain these items are held, the better. By holding inventory 'upstream', there is greater flexibility to redirect it to the point of need. Key to having this ability is a rapid and flexible transport system under the direct control of the SOF that cannot be 
redirected outside of the SOF chain without the acquiescence of the SOF itself. The US SOF has considerable air assets available to it through the 1st Special Operations Wing (SOW) which is committed to SOCOM exclusively, whilst UK SOF at present, relies upon earmarked aircraft from Nos. 7 and 47 Squadrons, RAF which are at the moment, theoretically outside of the SOF Commander's exclusive control.

Within this high risk, high impact area, the transport system must be able to effectively infiltrate and exfiltrate the environment encountered during the execution of these operations. It is insufficient merely to earmark aircraft, for the aircrew need to be properly trained as well.

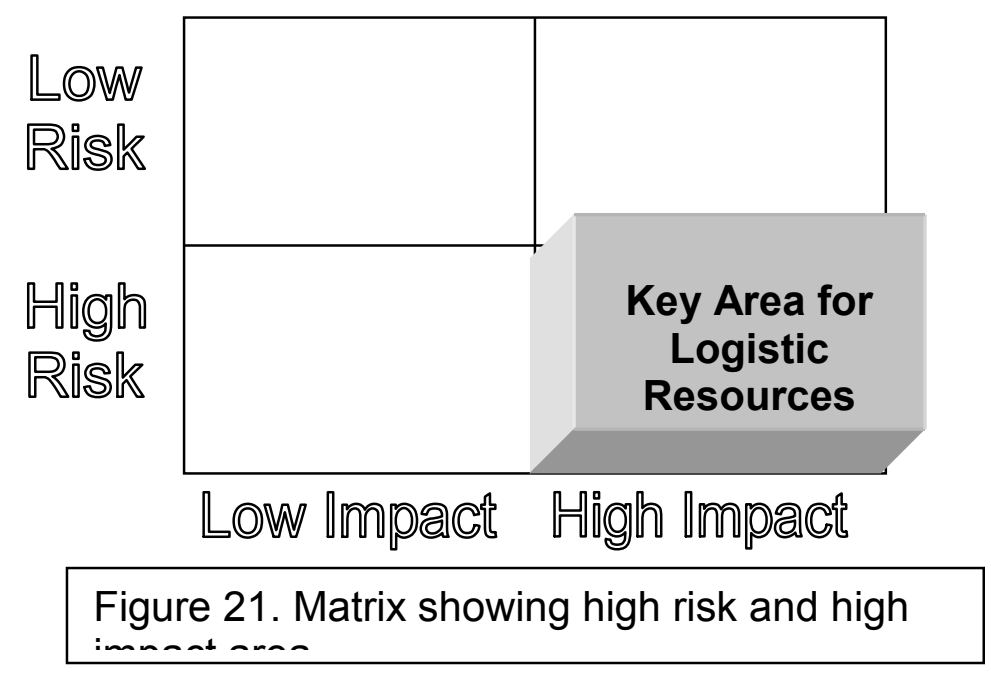

The high risk, low impact area encompasses those operations in which the risk of failure of the logistics system is relatively high but there is in fact a likelihood of a limited impact of that failure. Analysis of the case studies indicated that few missions fell within this area but those that did were of a low level and generally related to the 
Functional Quantitative Model. Technology levels were generally lower and there was often scope to be supported by much larger formations. To be effective in this area, the logistics system requires the following characteristics:

- Inventory levels can afford to be much lower and stocks can be reduced to minimal levels as the impact of the system failing is much less.

- Communications systems can be less comprehensive although contingency systems (and plans) are still required.

- Transport remains important, but parts of it can be outsourced to an outside provider as long as set levels of performance are met. This might mean a rear link operated by the Joint Force Logistic Component (JFLogC) of which SOF are just another user, with dedicated assets concentrated on operations in a more hostile environment.

- As inventory is reduced, the storage requirement decreases and use could be made of shared facilities to free up SOF assets elsewhere.

- Logistic planning is still required but can be more transactional in nature with perhaps less flexibility due to the reduced impact of the logistic plan failing.

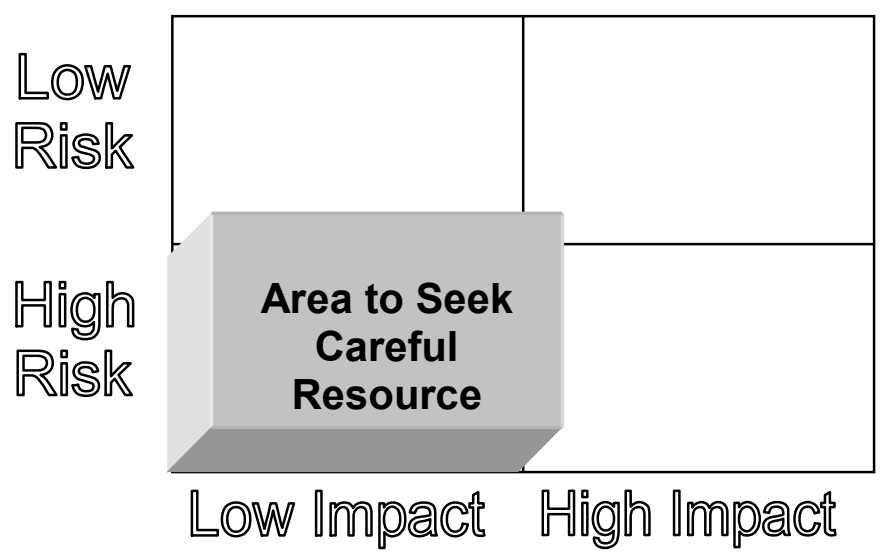

Figure 22. Matrix showing high risk and low 


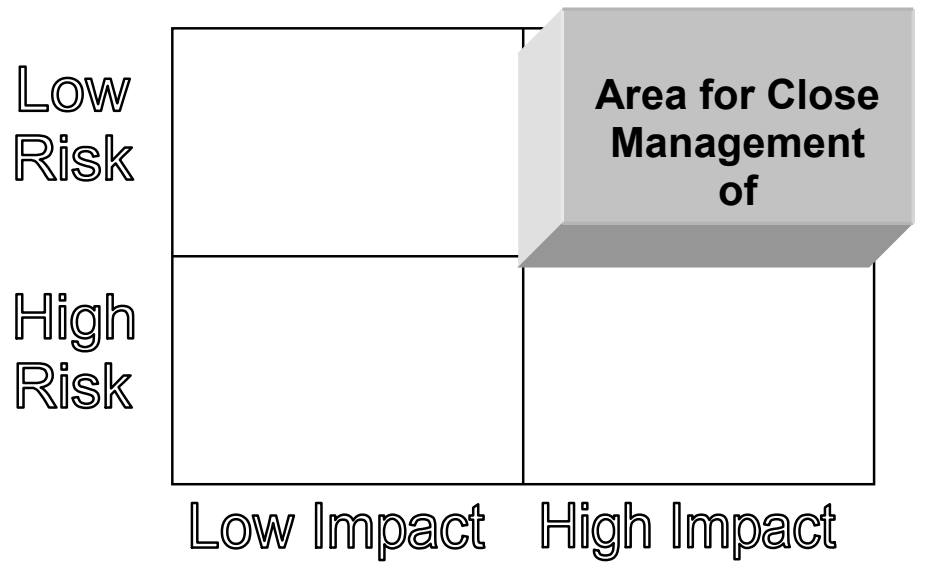

Figure 23. Matrix showing low risk and high

The low risk, high impact area is characterised by a higher level of operations that are commonly associated with low intensity conflict and carried out by predominantly Western style SOF. The high technology levels, combined with the diverse nature of the operations, makes the impact of logistic failure extremely high. Balanced against this is the risk minimisation benefits accrued through higher levels of training and commitment of the appropriate resources. To be effective the logistics system requires the following:

- Inventory levels need a degree of safety stock, as the impact of failure is high but a narrower range of items is likely to be required as there is a greater capacity to use common user items due to lower level of risk.

- A communications system that must be interoperable with conventional logistics systems to allow stock visibility.

- OPSEC must be preserved and should not be compromised for visibility.

- There should be a core transport capability, although additional elements can be called on from outside as required. 
- Logistic planning input is more important due to the high impact of logistics failure. Logistic planning requires full integration into the overall plan.

The final area to be considered is the low risk, low impact area. It is the area with the greatest scope for simplification and cost reduction, in order to strengthen the capability in other areas. The characteristics of this area are as follows:

- Inventory should be operated on a just-in-time basis. There should be minimal safety stocks procured and use should be made of local sourcing and commercial carriers to reduce necessary inventory levels.

- Communications should have commercial back-up systems to reduce costs.

- Transport should be acquired on and 'as required' basis from commercial sources with a limited core of operational vehicles.

- Storage requirements should be minimised and use made of hire facilities whenever possible.

- Logistics planning should be simplified, and rely on local purchase and the use of procurement cards to reduce logistics staff effort wherever possible.

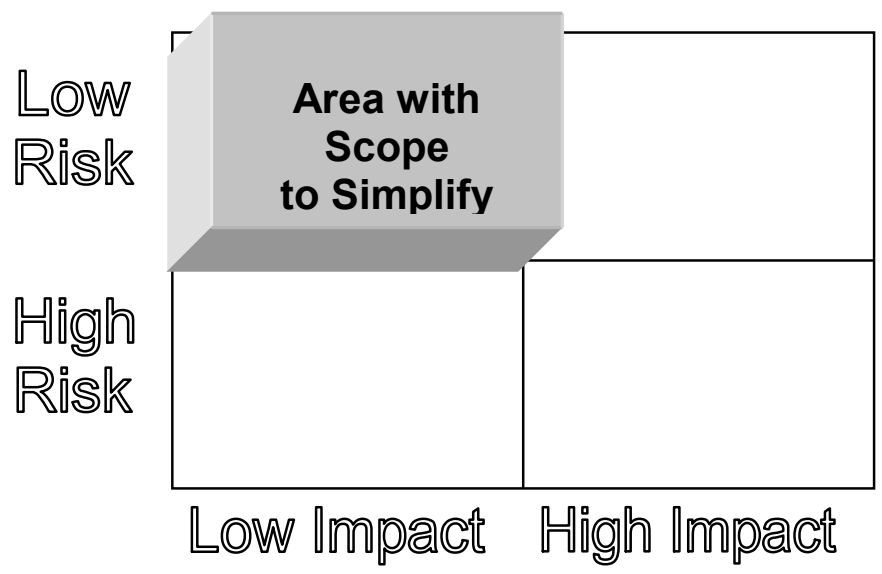

Figure 24. Matrix showing low risk and low 
Having looked at the strategies that can be derived from the application of a differentiation methodology such as the SOF Support Matrix, the final area the paper will examine is how best practice could be applied to improve these areas.

\section{Best Practice to Improve Logistic Performance}

Five key areas of best practice that characterised the improvement of the supply chain have been identified ${ }^{50}$ :

- Function to process - traditional organisations have been functional in design with each function having a clearly identified task. Best practice suggests that a cross-functional approach, with what was earlier termed mission focus, can produce better results. The idea must be to integrate logistics fully within the mission and not use it as a 'bolt on' extra at the end.

- Profit to performance - the growth of such systems as Resource Accounting and Budgeting is supposed to be output based and not cost based, as it was in the past. Whilst there is suspicion that the attempts to identify true costs are another way of cutting costs, the theory remains sound with a focus on output rather than cash input. Performance of a logistics system needs to be based on its performance and not its cost. SOF systems in particular, need to be recognised as having the requirement to meet the logistic imperatives and cannot be easily compared with other systems. Performance measurement of SOF systems need to take a holistic view of where they fit into the overall mission and not just crudely compared with other military systems.

- Product to customer - the development of a customer-supplier relationship within the SOF organisation is a useful way of identifying the stakeholders in 
logistic decision-making. With a firm customer focus rather than an obsession with its internal functional workings, logistics systems are better able to effectively target resources. The other side of this is the realisation that elements of SOF are required to be intelligent customers if they are to get the best out of the logistics system. Requirements need be specific and unambiguous to avoid any unnecessary over-insurance.

- Inventory to information - over-insurance through extensive inventory can be replaced through the better management of information. Replicated equipment can be replaced by more efficient distribution of smaller stocks and resources switched to the point of logistics vulnerability when required.

- Transaction to relationships - there needs to be an establishment of a set of clear relationships between SOF and its support, and a move away from simple transactional relationships. Its core capabilities must be fully integrated into the organisation and those elements that are outsourced need to be closely aligned to the SOF mission focus. Resources allocated to SOF for specific missions should come from elements well versed in the requirements of SOF and should have an established relationship with that organisation.

\section{Summary}

This paper has considered the role and purpose of special operations, which was drawn from a variety of sources. The key points were that Special Operations:

- Have a high political and military imperative

- Require specially trained and equipped forces

- Operate in an environment usually containing high political and military risk 
- Require high levels of military and political commitment to succeed

From this, it has proposed that Special Operations Forces are not a homogeneous entity but can be broadly distilled into three general models:

- Functional Quantitative Model (Russia)

- Comprehensive Qualitative Model (UK)

- Technical Functional Model (USA)

The paper highlighted the fact that SOF are under a wide variety of pressures, both internal and external, and how such organisations cope with these. Any logistics strategy needs to take this into account. These pressures, combined with logistics theory enable a set of logistic imperatives to be produced. In effect, these define the challenge of logistic support to SOF. These imperatives were:

- Self-sufficiency

- Demand pull

- Operational security

- Freedom of action

- Interoperability

- Accountability

In order to resolve this problem, a hypothesis was formulated that postulated that there was a link between the risk of logistic failure and the impact of that failure in terms of:

- The scale of the operation

- The role being undertaken 
- The model of SOF being followed

A number of case studies were utilised to test the hypothesis. Whilst the case studies utilised could not be conclusive, it was broadly indicated that there was a link between these factors. The solution to the problem is grounded in the need for the differentiation of strategy to ensure effective targeting of logistic resources. Different strategies for different areas of risk and impact indicate the optimum approach to balance cost effectiveness against operational success. These are summarised below.

Taking into account the key imperatives listed earlier it can be seen that, to be effective in the area of high-risk, high-impact operations, the logistic system must include:

- Levels of inventory consistent with the communication and transport capability of the force;

- Asset visibility with real-time transmission of demand with system redundancy ${ }^{51}$;

- Dedicated high-speed transport capability ${ }^{52}$ that is appropriate to the operating environment ${ }^{53}$;

- Storage requirements recognising different climatic scenarios;

- Integration of logistic needs with communication systems.

High-risk, low-impact operations encompass those operations where the risk of failure of the logistic system is relatively high, but there is limited impact from such failure. Key points are that:

- Inventory levels can afford to be much lower than other operations; 
- Communications system may be less comprehensive (but contingency systems are still required);

- Transport is important but could potentially be outsourced;

- Storage requirement is reduced.

Low-risk, high-impact scenarios is an area traditionally carried out by predominantly Western-style SOF. To be effective, the logistics system requires the following characteristics:

- Inventory levels must have a degree of safety stocks, although only a range of items is likely to be required;

- Stock visibility must be permitted through communications systems that can use conventional logistics systems - operational security must be preserved, not compromised due to logistic visibility;

- There is a need for a core transport capability;

- Logistic planning input requires full-integration into the overall plan.

Low-risk, low impact operations are where there is the greatest scope for simplification and cost reduction in order to strengthen the capability in other areas. Characteristics include:

- Inventory could be operated on a just-in-time basis with use made of local sourcing and commercial carriers to reduce inventory levels;

- Communications should have commercial back-up systems to reduce costs;

- Transport could be supplemented on an as-required basis from commercial hiresources;

- Storage requirements should be minimised; 
- Logistic planning should be simplified, for example using local purchase or procurement card.

These logistic strategies can be summarised in the diagram below:

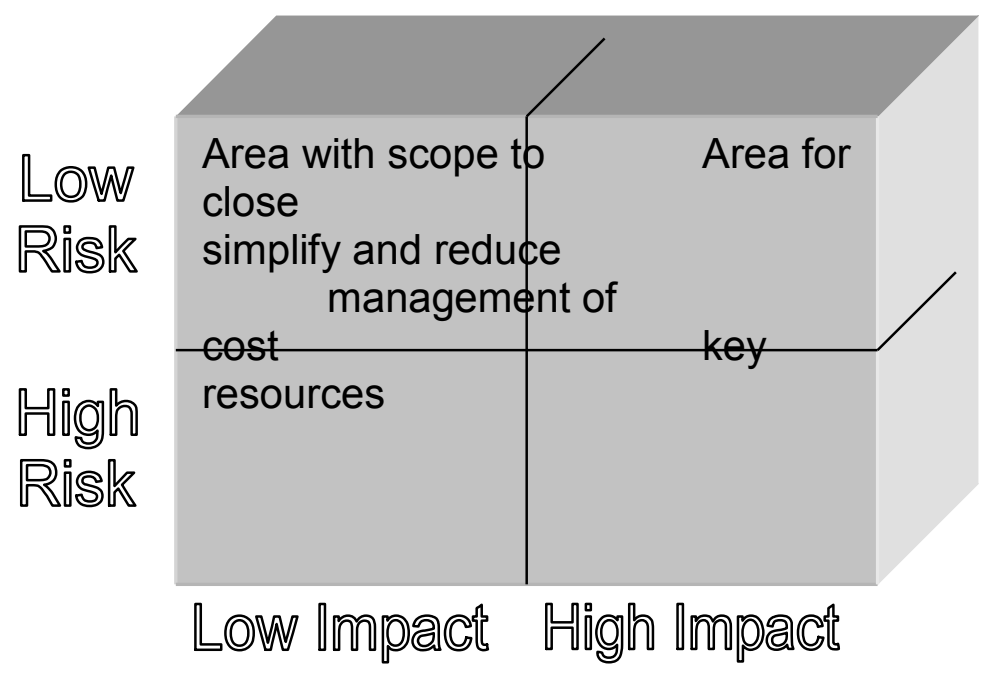

Figure 25. Matrix showing risk and impact

\section{Future Support to Special Operations Forces}

Before concluding, the paper will briefly look at how each of the logistic imperatives could be influenced by the development of technology.

- Self-sufficiency - improvements in self-sufficiency could be achieved through demand reduction technology. At the individual level, there are several key developments. Nanoscience ${ }^{54}$ technologies can reduce the weight of ballistic protection devices in addition to the production of ultra-light weight alternatives to existing equipment. Biomimetrics ${ }^{55}$ could enable the replication of food from local 
materials and vegetation and so reduce the food load for long-range operations, whilst technologies such as nutrition patches will allow individual endurance to improve. Sleep management techniques and technologies, such as those pioneered by B-2 Spirit crews when conducting missions over Serbia from the continental United States (CONUS) could enable individual soldiers to stay awake for over 72 hours without loss of performance. ${ }^{56}$ Sports technologies that pioneered the improvement of athletic performance can also improve fitness levels of SOF and so increase the ability to manage the loads that are required for a self-sufficient operation. A key area for technological development is that of power management, particularly for communications devices, all of which are extremely demanding on available resources. Future developments, such as selfgeneration, through routine movements such as walking, and micro fuel cells, can all reduce the combat load of SOF without loss of capability.

- OPSEC - the preservation of OPSEC can be enhanced through the development of stealth / masking technologies ${ }^{57}$, which can conceal logistic support assets, both visually and electronically. The ability to communicate effectively worldwide can also enable wide dispersal of assets, with no loss of co-ordination and control.

- Demand pull - the need for demand pull requires effective communication throughout the system. The growth of global ICT (Information and Communications Technology) associated with such areas as the Internet and mobile communications, enables effective global communications. When combined with rapid transport systems that are capable of penetrating hostile 
airspace, such as the MV-22 Osprey, cargo carrying unmanned aerial vehicles $(\mathrm{UAV})^{58}$, stand-off precision air drops ${ }^{59}$ and cargo carrying cruise missiles ${ }^{60}$, the risk to aircraft and air crew can be greatly reduced.

- Freedom of action - the ability to have complete visibility of support assets across a whole force can enable effective logistics input to the operational plan and enable the commander to have clearly-defined freedom of action. Development of multi-role equipment through modular production techniques allowing simple component exchange for additional capabilities ${ }^{61}$ and could reduce overall equipment requirements and so reduce logistic drag.

- Interoperability - the increased cost of research and development of new technology means that many programmes are collaborative, with the additional result of that there is often 'de facto' interoperability. Most SOF technology is driven by the United States, one of the few nations to be able to afford largescale SOF technology development. The dominant position of the United States in the area of air assets, means that NATO SOF must be able to operate with US technology, such as the 'HAVE QUICK' transmission modes. ${ }^{62}$ Technology enables standardisation through portals, that have developed commercially, to link new and legacy systems.

- Accountability - the need for accountability, for legal and political reasons, is enabled through the adaptation of technologies developed by law enforcement agencies, such as forensics and video technology. ICT allows better visibility than ever before, with technology such as bar coding and radio frequency 
technology improving the ability of the logistic system to stand up to any form of post-action scrutiny.

\section{Conclusion}

Special Operations Forces are not homogeneous and have developed due to a variety of influences, both internal and external. As a strategic asset, SOF are closely influenced by the politics of the nation and by the economic and cultural forces that define it. These external factors themselves can define the internal influences by dictating resources and roles. These influences often represent strengths such as the capability to operate claudestinely but also represent a source of weakness to the organisation. The ability to operate independently can create a culture of distrust of outside agencies that can lead to inefficiencies. It is important that any support strategy understands the influences that the SOF is under, both internally and externally.

There is a common framework in commercial logistics that is equally applicable to SOF logistics. The principles of the supply chain or support network with its elements of inventory, information etc. are just as relevant to SOF as to a commercial organisation. The application of commercial concepts, cannot however, be prescriptive and it must be recognised that there are different performance parameters to be considered. A commercial organisation is driven by satisfying stakeholders' expectations, which is generally manifested in financial terms. SOF stakeholders also require satisfaction, but this is rarely expressed in pure financial terms and is generally more intangible. 
SOF support is based on the concept of risk management. ${ }^{63}$ Special Operations balance the benefits of success against the risks of failure and this balance is the key to operational planning. The logistics strategy influences the scale of risk and ultimately the chance of success and so the performance of the system should be measured in terms of risk and impact over the usual financial indicators used in the management of a logistics system. The case studies support this view, with logistic failure considerations having a key role in several of the missions.

Any SOF support strategy must take the logistic imperatives into account if it is to effectively manage risk whilst attempting to efficiently manage resources. The logistic imperatives represent the factors that must be balanced to meet stakeholder requirements in the provision of support. They can be contradictory and so a compromise between the factors must be made to produce the most effective approach. Balancing these factors represents a 'trade-off' between the operational imperatives, which are demanding of resources, and the financial imperative, which aims to minimise expenditure.

The study of historical data can provide useful insights in order to evaluate the validity of a hypothesis. The interpretation of this data is essentially qualitative and requires skills of a historian rather than a statistician. However once a common approach to the study of the historical data is established, it becomes apparent that many historical principles are equally applicable even if clothed in different terminology, despite differences in specific detail. It is these general principles, which take account of the environment in which the events are set which are of 
value. It is meaningless to compare directly for example, Soviet operations in Levant in 1937 with Operation Desert Storm, but what can be undertaken is setting each in their historical context to derive the degree of prominence attached to logistics support for that operation. This cannot be done prescriptively, but needs to take account of the specific situation as it existed.

Having established that a degree of differentiation between scale, role and model exists, differentiated strategies can be formulated based on risk and impact management decisions using commercial best practice where applicable to derive key principles that can balance the logistic imperatives. The key must be to apply commercial logistics principles carefully in the context of each situation and not merely adopt commercial practice prescriptively. A strategy of differentiation is necessary to effectively target resources where they are required most.

The SOF Support Model can be used to inform the risk management decisionmaking by placing logistic plans into an appropriate context of risk and impact. By identifying areas of high risk and impact, logistics resources can be targeted towards the area where they would be of greatest benefit. In each differentiated area, a different balance of the logistic imperatives has to be found that enables a greater or lesser degree of commercial involvement depending upon the situation.

\footnotetext{
1 US Joint Chiefs of Staff. Doctrine for Joint Special Operations, JCS Publication 3-05, Washington, 1992, pp. $1-2$.

2 Paschall, R. Low Intensity Conflict: 2010, Brasseys, Washington, 1990, p. 8.

3 Cohen, E. Commandos and Politicians: Elite Military Units in Modern Democracies, Harvard Press, 1978, p. 17.

4 Piatkowski, K. 'Poland: Polish Special Forces in Search of a New Role' in The Journal of Slavic Military Studies, Vol. 11, No. 3, 1998, pp. 105 - 127.

5 The operation to retake Grenada in 1983.

6 Panama, 1989.

7 Xavier Raufer. 'Gray Areas: A New Security Threat' in Political Warfare: Intelligence, Active Measures and Terrorism, Report No. 20, Spring 1992. Quoted in R J Bunker. Five Dimensional
} 
Warfighting: Can the Army After Next be Defeated through Complex Concepts and Technologies?, Strategic Studies Institute, US Army War College, Carlisle Barracks, PA, March 1998.

${ }^{8}$ Krulak, General Charles. 'World without symmetry - terrorism' in Navy Times, 22 September 1997, pp. $5-7$.

${ }_{9}$ Op Cit. Bunker, 1998, pp. 5 - 10. The dimensions are length, breadth, height and time.

${ }^{10}$ Cyberspace is most often associated with the use of computers and the Internet / World Wide Web, and while this is perfectly true, it is increasingly being used as a term to represent that area beyond human senses that allows space and time to be dissolved. Op Cit. Bunker, 1998.

11 Gray, C. 'Handfuls of heroes on desperate ventures: When do Special Operations succeed?' in Parameters, Spring 1999, pp. 2 - 24, p. 5.

12 Ibid. p. 4. Such an operation was illegal under the Geneva Convention Additional Protocol II 1977 on civilian immunity from attack.

${ }^{13}$ Karl von Clausewitz, On War (1832) quoted in Royle, Trevor. Collins Dictionary of Military Quotations, HarperCollins, Glasgow, 1991, p. 14.

${ }^{14}$ Eagle Claw was the codename for the operation overall, while the actual embassy assault went by the codename Rice Bowl.

${ }^{15}$ Quoted in Regan, G. Great Military Blunders, Macmillan, London, 2000, p. 53.

${ }^{16}$ Brandenburgers were the SOF element of Nazi Germany's military intelligence, the Abwehr, under Admiral Canaris.

${ }^{17}$ McKevitt, David and Lawton, Alan. Public Sector Management, Sage, 1994, London, pp. $14-15$.

18 Marley, A D. 'Special Operations in Conflict Resolution' in Small Wars and Insurgencies, Vol. 7, No. 1, 1996, pp. $35-40$.

${ }^{19}$ For example, the US Rangers were forced to scrap their M-151 Jeeps when the HMMWV was introduced for budget reasons. They have now had to buy UK Land Rovers because the HMMWV does not fit inside a CH-47 helicopter or CV-22 aircraft, whereas the M-151 did. Author's conversation with Chief J4 SOCACOM, JTFEX-99.

20 The interception and shooting of three IRA terrorists in Gibraltar, 1988. Geraghty, T. Who Dares Wins, Little, Brown \& Company, 1992, London, pp. 282 - 317.

${ }^{21}$ Members of the Military Assistance Command Vietnam - Studies and Observation Group were used in the Phoenix programme to assassinate Viet Cong sympathisers within the South Vietnamese political system. They are immortalised in many ways by Martin Sheen's character in the film,

Apocalypse Now. Asprey, R B. War in the Shadows, William Morrow, 1994, pp. $909-912$ and Elliot, P. Vietnam: Conflict and Controversy, Arms \& Armour Press, London, 1996, pp. $106-9$.

22 The winged dagger is, more correctly, a winged sword of Damocles, but has come to be known as a winged dagger in the popular press. James, M. Born in the Desert, Collins, London, 1945 (republished by Greenhill, 1991).

${ }^{23}$ This is also termed 'think-evil' and seems to be more of a Western trait, although it has been detected in some parts of the Spetnaz (Schofield, C. Inside the Soviet Army, Headline, 1991, pp. 198 $-201)$.

${ }^{24}$ Etzioni, A. The Moral Dimension: Towards a New Economics, New York Free Press, 1988, pp. 83 85.

${ }^{25}$ Derived from Porter, $\mathrm{M}$ in Johnson, $\mathrm{G}$ and Scholes, K. Exploring Corporate Strategy, Prentice Hall, Hemel Hempstead, 1999, pp. $115-120$.

${ }^{26}$ Sometimes referred to as 'factory-to-foxhole'.

${ }^{27}$ Bowersox, D J and Closs D J. Logistical Management, McGraw Hill, New York, 1996, pp. 25 - 29.

${ }^{28}$ Slack, N et al. Operations Management, Financial Times / Prentice Hall, London, 1997, p. 432.

${ }^{29}$ This is the NATO term. The UK equivalent is Forward Mounting Base (FMB).

${ }^{30} \mathrm{HMS}$ Antrim acted as the ISB for UK SOF in Operation Paraquat, the operation to recapture South Georgia in 1982. Op Cit. Geraghty, 1992, pp. $84-88$.

31 Op Cit. Bowersox and Closs, 1996, p. 187.

32 Those activities or actions that are sponsored or conducted by SOF in such a way as to maximise secrecy or concealment. It differs from covert operations in that emphasis is placed on concealment of the operation rather than on concealment of the identity of the sponsor.

${ }^{33} \mathrm{~A}$ process of analysing friendly actions in relation to military operations to minimise the risks to the operation from enemy activity.

${ }^{34}$ For example, it was believed that a number of US Navy SEALs were drowned during Operation Urgent Fury (Grenada) after a water parachute jump due to severe overloading with over $150 \mathrm{~kg}$ of equipment. Marquis, S. Unconventional Warfare: Rebuilding US Special Operations Forces, The Brookings Institute, 1997, pp. 96 - 97. 
${ }^{35}$ SAS Jeeps in the Western Desert during WW2 suffered severe overloading problems when compared to LRDG patrols who opted to use the $30 \mathrm{CWT}$ Chevrolet trucks with their greater capacity. Swinson, A. The Raiders: Desert Strike Force, Pan Ballantine, 1968, pp. 16 - 18.

${ }^{36}$ B Squadron 22 SAS was severely limited during Operation Granby (the Gulf War 1991) by the availability of equipment which had gone to $A$ and $D$ Squadrons deployed earlier in the operation. Weale, A. Secret Warfare, Hodder and Stoughton, 1997, pp. $268-270$.

${ }^{37}$ Hogg, I V \& Weeks, J. Military Small Arms of the Twentieth Century, Arms and Armour Press, 1993, pp. $335-336$.

38 Spetsnaz operations in Afghanistan were large in terms of manpower (over 200 personnel were not uncommon) but rarely incorporated the wide range of assets and capabilities usually found at the Task Force level. David C Isby in Burgess, W. (ed) Inside Spetsnaz: Soviet Special Operations - A Critical Analysis, Harper Collins, 1990, pp. $203-221$.

${ }^{39} \mathrm{~A}$ process which aims to conserve the fighting potential of the force by countering the wider threat to its elements from adversaries, natural and human hazards and fratricide. Newman, $\mathrm{N} \mathrm{J}$.

Asymmetric Threats to British Military Intervention Operations, RUSI, 2000, Whitehall Paper No. 49, p. 90.

40 Operation Eagle Claw / Rice Bowl was scrubbed at Desert One but the strict restrictions on incurring casualties made the operation unworkable. Holloway Special Operations Review Group quoted in Op Cit. Marquis, S. (1997), pp. $72-82$.

${ }^{41}$ SAS patrols in Borneo had a restriction of $30 \mathrm{lbs}$ of equipment placed on them in order for them to remain agile in cross-border operations. Kemp, A. SAS: Savage Wars of Peace, John Murray

Publishers, 1994, pp. $64-5$.

42 Few nations possess effective air penetration platforms capable of delivering items in this manner. Only the United States possesses a significant capability with the MC-130 Combat Talon for longrange delivery. Such delivery over shorter ranges is still carried out by helicopters or tilt rotorcraft such as the MH-53 Pave Low or MV-22 Osprey.

${ }^{43}$ The limousine carried by Israeli Commandos at Entebbe almost caused a breach of security when a vehicle could not be sourced from official sources and one had to be purchased on the open market, a possible indication of an up-and-coming operation. McRaven, W H. Spec Ops: Case Studies in Special Operations, Presidio Press, 1995, pp. $349-50$.

${ }^{44}$ Many of the LRDG supply dumps in the desert were only recorded at patrol level for security reasons which unfortunately denied their use to other task elements. Op Cit. Swinson, A. (1968), pp. $15-16$.

${ }^{45}$ Christopher, Prof M. Logistics and Supply Chain Management, Financial Times / Pitman Publishing, 1998, 2nd Ed, pp. 56 - 7.

${ }^{46}$ This differentiation is carried out by such means as the 'Pareto' or ABC analysis. Op Cit. Christopher (1998) pp. $57-60$.

${ }^{47}$ The provision of an activity from outside the organisation. Op Cit. Christopher (1998) p. 15.

48 The ability for management at the various levels of command to know the location, quantity and condition of any or all of a defined range of equipments and commodities and where appropriate the ultimate use to which those commodities are put. Ministry of Defence. Logistics, ADP (Army Doctrine Publications) Vol. 3, 1996, p. xix.

${ }^{49}$ As noted earlier, LRDG patrols used caches of supplies that were often not known to other elements that could have used them more effectively.

50 Op Cit. Christopher (1998) pp. 260 - 264.

${ }^{51}$ Many SOF rely on several layers of communication. Many primary systems are UHF Tactical Satellite (TACSAT) with a High Frequency back up. Additional means include emergency location systems with air-to-ground communications. Pengelly, R. 'Forward Air Controllers or Flag Wavers? Seeking Common Ground' in Jane's International Defence Review, September 1999, pp. 56 - 58.

52 This includes both ground and air vehicles, as flying may be impossible in some situations.

53 The RH-53s used in Operation Eagle Claw were modified minesweeping helicopters that were not fitted with sand filters and suffered severe sand ingestion on the flight into Iran.

${ }^{54}$ Science conducted at the molecular level.

${ }^{55}$ Adapting natural processes to practical applications.

56 Haslam \& Abraham in Belenky, G. (ed) Contemporary Studies in Combat Psychiatry, Greenwood

Press, 1987, pp. $167-184$.

57 Op Cit. Bunker (1998) pp. 7 - 9. 
58 Op Cit. Paschall (1990) pp. $57-69$ and Hewish, M. 'US Technology aims to ease FAC task' in Jane's International Defence Review, September 1999, pp. 62 - 63 and 'Coming soon: attack of the killer UAVs' in Jane's International Defence Review, September 1999, pp. 30 - 32.

${ }^{59}$ Such as the Containerised Air Delivery System (CADS) used by UK and US forces. Jane's Information Group, Jane's Counter Terrorist Equipment, 1997, p. 214.

60 Op Cit. Paschall (1990) pp. 48 - 9.

61 Van Hoek, R and Weken, H A M. 'The Impact of Modular Production on the Dynamics of Supply Chains' in International Journal of Logistics Management, Vol. 9 No. 2, 1998, pp. 35 - 49.

62 Op Cit. Pengelly (1999) pp. $57-58$.

${ }^{63}$ A similar risk and impact process is carried out by Corporate Insurers to determine premiums. Author's conversation with Aon Group Limited Risk Assessor. 